\title{
Introduction to Topological Quantum Computation
}

\author{
V.T. Lahtinen ${ }^{1}$, J.K. Pachos ${ }^{2, *}$, \\ 1 Freie Universität Berlin, Arnimallee 14, 14195 Berlin, Germany \\ 2 School of Physics and Astronomy, University of Leeds, Leeds, LS2 9JT, United Kingdom \\ *J.K.Pachos@leeds.ac.uk
}

November 19, 2019

\begin{abstract}
This review presents an entry-level introduction to topological quantum computation - quantum computing with anyons. This approach is inherently resilient against errors, thus promising to overcome one of the main obstacles for the realisation of quantum computers. We introduce the concept of anyon models and review the literature on condensed matter systems where anyons can emerge. Then we discuss the general steps how to use anyons to encode and process quantum information, as well as discuss various ways topological protection might fail. Finally, these abstract concepts are demonstrated in the concrete system of Kitaev's topological nanowire. This model supports localised Majorana zero modes - the simplest and experimentally most tractable types of non-Abelian anyons - and it describes the low-energy physics of several experimentally relevant settings.
\end{abstract}

\section{Contents}

1 Introduction 2

1.1 Topology, stability and anyons

1.2 Anyons in condensed matter systems 5

2 Anyon models

2.1 Fusion channels - Decoherence-free subspaces 8

2.2 Statistical quantum evolutions 9

2.3 Example 1: Fibonacci anyons 11

2.4 Example 2: Ising anyons 12

3 Quantum computation with anyons 13

3.1 Initialization of a topological quantum computer 14

3.2 Quantum gates - Braiding anyons

3.3 Measurements - Fusing anyons 16

3.4 Possible error sources 17

4 Manifestations of anyons in microscopic many-body systems 18

4.1 Ground state degeneracy and Berry phases 18

4.2 Topological degeneracy and entanglement entropy 20

5 Majorana zero modes in a superconducting nanowire 21 
5.1 Kitaev's toy model for a topological nanowire

5.2 Quantum computation in nanowire arrays 23

5.2.1 The Majorana qubit 23

5.2.2 Manipulating the Majorana qubit 25

5.2.3 Challenges with Majorana-based topological quantum computation 25

6 Outlook 27

References

\section{Introduction}

Topological quantum computation is an approach to storing and manipulating quantum information that employs exotic quasiparticles, called anyons. Anyons are interesting on their own right in fundamental physics, as they generalise the statistics of the commonly known bosons and fermions. Due to this exotic statistical behaviour they exhibit quantum evolutions that are described by topology, i.e. they are abstracted from geometrical details. This topological behaviour provides a much desired resilience of the constructed evolutions to control errors and perturbations. While from the first sight anyons appear to be an over-complicated method for performing quantum computation, they are profoundly linked to quantum error correction [1], the algorithmic means we have in dealing with errors during quantum computation. In a sense, anyonic quantum computers implement quantum errorcorrection at the hardware level, thus becoming resilient to control errors and erroneous perturbations. This has augmented topological quantum computation from a niche field of research to a methodology that permeates much of the research efforts in realising fault-tolerant quantum computation.

\subsection{Topology, stability and anyons}

In mathematics, topology is the study of the global properties of manifolds that are insensitive to local smooth deformations. The overused, but still illustrative example is the topological equivalence between a donut and a coffee cup. Regardless of the local details that give them rather different everyday practicality, both are mathematically described by genus one manifolds meaning that there is a single hole in both. Small smooth deformations, such as taking a bite on the side of the donut or chipping away a piece of the cup will change the object locally, but the topology remains unchanged. Only global violent deformations, such as cutting the donut in half or breaking the cup handle, will change the topology by removing the hole.

In real world small deformations matter though. Quite spectacular salesmanship is required to sell a donut from which someone has already taken a bite. Something similar occurs also in quantum mechanics. To store and evolve a pure quantum state coherently, one must take exceptional care that no outside noise interferes and that the evolution is precisely the desired one. This is the key fundamental challenge in quantum computation: to robustly store quantum states for long times and evolve them according to specific quantum gates. Were quantum information encoded in topological properties of matter though, and were the quantum gates dependent only on the topology of the evolutions, then both should be inherently protected from local deformations such as erroneous perturbations or 
error in implementing quantum gates. Such topological quantum computation would exhibit inherent hardware-level stability that ideally would make elaborate schemes of quantum error-correction redundant.

This idea was first floated by Kitaev in connection to surface codes for quantum error correction [1, 2]. He realized that certain codes could be viewed as spin lattice models, where the elementary excitations are anyons - quasiparticles with statistics interpolating between those of bosons and fermions [3]. By manipulating these excitations, quantum states could be encoded in the global properties of the system and manipulated by transporting the anyons along non-contractible paths. The local nature of the paths would be irrelevant - any two paths that were topologically equivalent implemented the same quantum gate. This insight put the study of anyons at the center of topological quantum computation. Importantly, it gave significant renewed incentive to condensed-matter physicists to look for realistic systems that could give rise to them.

The reason why anyons can exist in general can be traced back to the simple, but far reaching realization that local physics should remain unchanged when two identical particles are exchanged. In three spatial dimensions (3D) it dictates that only bosons and fermions can exist as point-like particles. A wave function describing the system of both particles acquires $a+1$ or a -1 phase, respectively, whenever they are exchanged. However, when one goes down to two spatial dimensions (2D), perhaps somewhat counter-intuitively as the number of degrees of freedom is reduced, a much richer variety of statistical behaviour is allowed. In addition to bosonic and fermionic exchange statistics, arbitrary phase factors, or even non-trivial unitary evolutions, can be obtained when two particles are exchanged. Hence, anyons can in principle exist.

The fundamental difference between 2D and 3D is the topology of space-time evolutions of pointlike particles. Consider the exchange processes of two particles illustrated in Figure 1 . In three dimensions the path $\lambda_{2}$ drawn by the encircling particle is always continuously deformable to the path $\lambda_{1}$ that does not encircle the other particle. Such a deformation of the trajectory is allowed as we consider the particles to be non-interacting, and thus their relative distance is irrelevant in this evolution. This loop, in turn, is fully contractible to a point, which means that the wave function of the system must satisfy

$$
\text { 3D : } \quad\left|\Psi\left(\lambda_{2}\right)\right\rangle=\left|\Psi\left(\lambda_{1}\right)\right\rangle=|\Psi(0)\rangle .
$$

As one particle encircles the other twice, the evolution of the system can be represented by the exchange operator $R$ such that $\left|\Psi\left(\lambda_{2}\right)\right\rangle=R^{2}|\Psi(0)\rangle$. The contractibility of the loop requires that $R^{2}=1$, which has only the solutions $R= \pm 1$ that correspond to the exchange statistics of either bosons or fermions. This means that the order and the orientation of the exchanges are not relevant and the statistics of point-like particles in 3D are mathematically described by the permutation group.

This contrasts with $2 \mathrm{D}$, where the path $\lambda_{2}$ is no longer continuously deformable (the path is not allowed to cross the encircled particle) to the fully contractible path $\lambda_{1}$. This means that the final state $\left|\Psi\left(\lambda_{2}\right)\right\rangle$ no longer needs to equal the initial state $|\Psi(0)\rangle$

$$
\text { 2D : } \quad\left|\Psi\left(\lambda_{2}\right)\right\rangle \neq\left|\Psi\left(\lambda_{1}\right)\right\rangle=|\Psi(0)\rangle .
$$

Hence the exchange operator $R$ is no longer constrained to square to identity either. Instead, it can be represented by a complex phase, or even a unitary matrix. In the first case the anyons are called Abelian anyons due to their exchange operators commuting, while in the latter case the anyons are referred to as non-Abelian anyons. Since one no longer demands $R=R^{-1}$, the order and orientation of the exchanges are physical and the only constraints on the exchange operator $R$ are given by consistency conditions for distinct evolutions. These derive from a mathematical structure known as the braid group, which describes all topologically distinct evolutions of point-like particles in two spatial dimensions. It is 

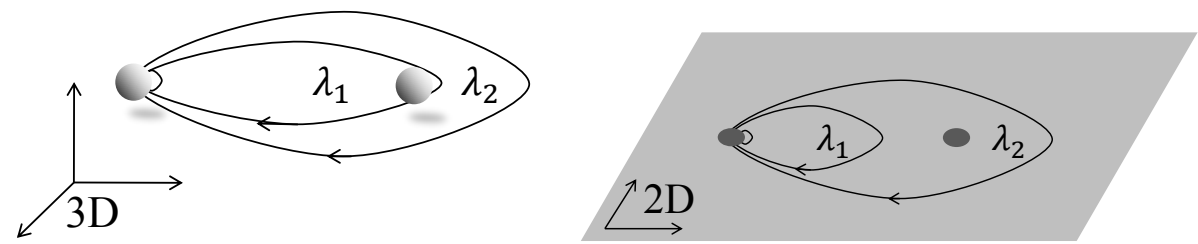

Figure 1: Exchange statistics in 2D vs. 3D. In 3D the path $\lambda_{2}$ describing two particle exchanges is continuously deformable to $\lambda_{1}$ that encloses no particles, which in turn is contractible to a point. In $2 \mathrm{D}$, however, the paths $\lambda_{2}$ and $\lambda_{1}$ are topologically inequivalent since the path can not be deformed through the particle, while $\lambda_{1}$ is still contractible to a point. Hence, different quantum mechanical evolutions can be assigned to each of the topologically different types of exchanges.

this description of the 2D statistics by the braid group, instead of the permutation group, that allows anyons to exist.

While the possibility of something to exist is not equivalent to actually existing, this simple analysis gives the key hint for where to look for anyons - systems that are 2D. As we are living in a 3D world (at least what comes to our every day energy scales), no genuine 2D systems exist. Nevertheless, many systems can be constrained to exhibit effective 2D behavior, such as electron gases at 2D interfaces of 3D materials, isolated sheets of atoms such as graphene or 2D optical lattices of cold atoms. One should always keep in mind though that 2D only enables anyons to exist, but by no means guarantees that. In fact, the emergence of anyons requires quite special conditions, such as exotic interactions, that can be illustrated using an intuitive toy model for anyons [4]. Consider a composite particle that consists of a magnetic flux $\Phi$ confined inside a small solenoid and a ring of electric charge $q$ around it, as illustrated in Figure 2. If one such quasiparticle encircles the other, then its charge $q$ goes around the flux $\Phi$, and vice versa. Due to the celebrated Aharonov-Bohm effect [5], the wave function of the system acquires a phase factor $e^{2 i q \Phi}$, even if there is no direct interaction between the quasiparticles. Since all the magnetic flux is confined to the solenoid, this phase factor does not depend in the local details of the path. It depends only on the number of times one particle circulates the other, which makes it topological in nature. These observations imply that if

$$
2 q \Phi \neq 2 \pi n, \quad n=1,2, \ldots,
$$

then the wave function will evolve exactly in the same way as a system of two anyons with exchange statistics described by $R=e^{i q \Phi}$. This happens if the charge or the flux is fractionalised, i.e. $q \neq n$ (in units of the electron charge) or $\Phi \neq 2 \pi n$, respectively. While this is a toy picture, it demonstrates the intimate connection between anyons and fractionalisation. It suggests that strongly interacting systems are required for anyons to emerge as localized collective quasiparticle states of the fundamental particles such as electrons or spin moments.

The study of the strongly correlated system that support anyons is the subject of extensive condensed matter literature. In this review we adopt a point of view that anyons exist and that they are completely decoupled from the microscopic physics that give rise to them. This enables to present how quantum information is encoded and processed by purely topological means. However, before proceeding with this main topic, we give a brief literature review on the numerous physical systems that support or are proposed to support anyons. 


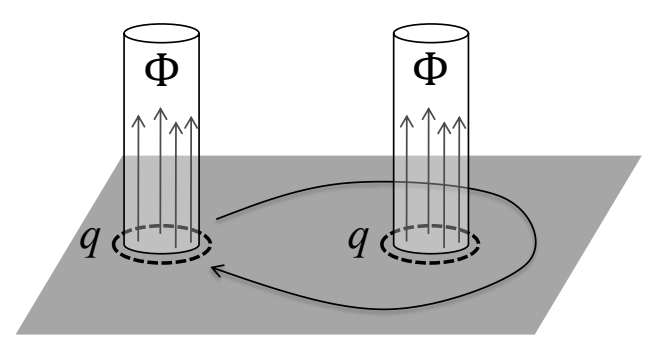

Figure 2: Toy model for anyons as charge-flux composites where magnetic flux $\Phi$ confined to a tube that is encircled by a ring of electric charge $q$. When one such composite object moves around the other, its charge (flux) circulates the flux (charge) of the other anyon. The Aharonov-Bohm effect gives rise to the complex phase $e^{2 i q \Phi}$, which describes the mutual statistics of the composite objects. For $2 q \Phi \neq 2 \pi$ they are Abelian anyons.

\subsection{Anyons in condensed matter systems}

Nowadays it is impossible to talk of distinct phases of matter without talking about topology. While the energy spectra of gapped free-fermion materials might look alike, their electronic wave functions themselves can be topologically distinct. It is possible to characterise them by topological winding numbers that, based on the symmetries present, enable to classify how many distinct states of matter there can be [6, 7]. In such systems the topological classification is only defined given that the protection symmetries (e.g. time-reversal, charge conjugation [8] or point-group symmetries arising from the underlying crystal lattice [9, 10, 11, 12]) are respected. These states of matter are usually referred to as symmetry-protected topological (SPT) states [13, 14, 15]. While topology in such states can give rise to interesting phenomena, such as protected surface currents, SPT states do not in general support anyons. This is a direct consequence of the fact that entanglement in such states is short-ranged and does not exhibit the characteristic subleading correction of topological entanglement entropy [16, 17, 18]. There is an intriguing exception to this rule though. Topological superconductors are also topological insulators, but due to the particle-hole symmetry, localized zero energy modes are Majorana (real) fermions that can be viewed as fractionalized halves of complex fermion modes [19]. For all practical purposes from the perspective of quantum computation, Majorana modes behave like non-Abelian anyons [20], but they are also the only kind of anyonic quasiparticles that can emerge in free-fermion systems. Unfortunately, while being the most experimentally accessible ones, they are not universal for quantum computation by purely topological means, as will be discussed later.

This contrasts with intrinsic topological order that does not require any symmetries to be present. These phases are characterised by their ability to support anyons. Some of these anyons are universal for quantum computation by purely topological means, and exhibit fractionalization beyond Majorana modes. They also exhibit long-range entanglement that gives rise to topological entanglement entropy [16, 17, 18] and ground state degeneracy that depends on the topology of the manifold the system is defined on [21] (for instance, the ground state degeneracy is different on a sphere and on a torus). The most studied systems known to support intrinsic topological order are fractional quantum Hall states and their bosonic cousins known as spin liquids. Thus the condensed matter systems that support anyons can be roughly divided into three classes: (i) Strongly correlated electron gases under strong magnetic fields that support fractional quantum Hall states, (ii) collective states of strongly interacting spins giving rise to spin liquid states and (iii) topological superconductors and in particular their engineered variants in topological insulator heterostructures. 
(i) Fractional quantum Hall states: Fractional quantum Hall $(\mathrm{FQH})$ states occur when very cold electron gases are subjected to high magnetic fields. In such states the electrons form Landau levels, making the system a highly degenerate insulator. Since the electron motion is frozen out, interactions between the electrons become significant. Due to the interactions the gapped ground states at different magnetic fields can be described by a non-integer filling fraction $v$ - the number of electrons per flux quantum, i.e. fractionalization occurs. While the bulk of the 2D system is insulating, experimentally such states are most easily detected by measuring the off-diagonal conductance that exhibits characteristic plateaus corresponding to different filling fractions [22, 23]. Every plateau is a distinct phase of intrinsic topological matter, characterized by the quantized conductance that is proportional to the topological invariant characterizing the state [24].

The nature of these states and the anyons they support is understood via trial wave functions. Such wave functions were first proposed by Laughlin to predict that the $v=1 / 3$ state supports fractionalized quasiparticles that behave as Abelian anyons [25]. While the direct probing of the anyons via their exchange statistics remains elusive, the charge fractionalization has been experimentally confirmed, thus strongly supporting the existence of anyons [26, 27]. Employing the intricate connection to conformal field theory, numerous further trial wave functions have been proposed to describe other filling fractions seen in the experiments. From the point of view of quantum computation, of particular interest is the $v=5 / 2$ case. It has been proposed that at this filling fraction the system is described by the Moore-Read state that supports the simplest non-Abelian anyons - the Ising anyons [28]. The predicted charge fractionalization has also been confirmed, but again direct probing of the anyons has remained elusive [30]. For most practical purposes Ising anyons are equivalent to Majorana modes and hence they are not universal for quantum computation. For universality one needs more complex Fibonacci anyons. These are expected to emerge in the very fragile $v=12 / 5$ filling fraction described by the Read-Rezayi state [29]. For a comprehensive account of topological quantum computation in FQH states, we refer the interested reader to [31].

It has also been proposed that FQH states can also emerge in topological insulators when they are subjected to similar conditions, i.e. strong magnetic fields, strong interactions and fractional filling [32]. While such fractional topological (Chern) insulators are an intriguing alternative, it is currently unclear whether these conditions can ever be achieved in crystalline materials.

(ii) Spin liquids: When strong Coulombic interactions localize electrons in a lattice configuration, their kinetic degrees of freedom are frozen out in a Mott insulating state. However, the electron spins still interact and can form collective states that exhibit topological order. Such states are known as topological spin liquids [33]. The study of these systems roughly follows two distinct routes. The bottom-up route is to study physically most common spin-spin interactions, which usually are of Heisenberg type, on distinct lattices. As such systems rarely lend themselves to analytic treatment, one employs mean-field theory to understand what phases could exist [34] and relies on state-ofthe-art numerics to verify the predictions. Convincing numerical evidence has been obtained that topological spin liquids that support Abelian anyons do exist on several frustrated 2D lattices (e.g. triangular or Kagome lattices) [35, 36, 37, 38, 39]. The top-down route is to write down idealized spin lattice models that support a given topological phase. The canonical model of this type is Kitaev's honeycomb model [40] that supports Ising anyons akin to the Moore-Read state. The model is exactly solvable, which enables the emergent anyons to be studied in detail, but the fine-tuning required for the exact solvability also means that it is unlikely to appear in nature. However, certain iridate compounds can be described by a perturbed version of the model [41,42]. Indeed, neutron-scattering experiments have provided initial evidence for spin liquid states in these materials [43].

Generalizations of the honeycomb model exist both in 2D and 3D [44, 45]. As these systems all follow the same construction, this family of models is still the only analytically tractable framework 
for spin liquids. In principle, spin liquids supporting any types of anyons can be defined on lattices via the quantum-double [2] or the Levin-Wen [46] construction, but these require replacing actual spins with more generic local degrees of freedom subject to rather unphysical constraints and involve many-body interactions. A celebrated example of the quantum-double construction is the so called Toric Code that supports Abelian anyons. The Toric Code is the simplest example of a topological surface code and it features heavily in relation to quantum memories and error correction.

(iii) Topological superconductors in heterostructures: Since the seminal work by Read and Green [47], it has been known that if the pairing in a 2D superconductor is so-called $p$-wave type, i.e. timereversal is broken by the pairing term, then vortices in such systems bind Majorana modes. While actual materials exhibiting such pairing are yet to be found (though strontium ruthenate is strongly believed to be one) it was realized that qualitatively same physics could occur when a topological insulator [48], a spin-orbit coupled semiconductor [49, 50], a chain of magnetic atoms [51, 52] or half-metals [53, 54] is placed in the proximity of a regular $s$-wave superconductor. In other words, the combination of physics from both systems would realize effective $p$-wave superconductivity at the interface. In particular, following an early proposal by Kitaev [55], wires made of these materials and deposited on top of a superconductor were predicted to host Majorana modes at their ends, which could be probed through simple conductance measurements [56, 57]. While the explicit verification of their braiding properties is yet to be carried out, several experiments on microscopically distinct setups strongly support the existence of Majorana modes [58, 59, 60, 61, 62]. These topological nanowire heterostructures are the most prominent candidate to experimentally test the key building blocks of topological protection and implementation of topological gates via braiding. For a comprehensive review of Majorana zero modes in solid-state systems, see e.g. [63, 64, 65].

(iii) Quantum simulations of anyonic systems: In addition to looking anyons in materials, much progress has been made to simulate such physics with cold atoms in optical lattices. Time-reversal symmetry broken Chern insulators [66, 67] have been realized and it is hoped that these systems can be pushed towards fractionalized conditions that support anyons. Furthermore, Kitaev's honeycomb model has been proposed to be realized with cold atoms [68] as have various cold atom [69, 70, 71] and cavity arrays [72] variants of topological nanowires that host Majorana modes. Alternatively, well controlled photonic quantum simulators have also been employed to probe the statistical properties of the Toric Code anyons [73] or of Majorana modes [74].

\section{Anyon models}

From now on we adopt a perspective that anyons exist and focus on explaining what different types of anyons there can be and what are their defining properties. Furthermore, for the time being, we assume that the microscopic details of the system that give rise to the anyons can be completely neglected and the low-energy dynamics can be described in terms of only the anyons. Under these assumptions the possible evolutions are limited to three simple scenarios:

1. Anyons can be created or annihilated in pairwise fashion.

2. Anyons can be fused to form other types of anyons.

3. Anyons can be exchanged adiabatically.

The formal framework capturing these properties in a unified fashion goes under the name of a topological quantum field theory [75]. From the point of view of topological quantum computation, most 
of the details of this rigorous mathematical description can be omitted. As a result, only a minimal set of data is required to specify the properties of the anyons corresponding to a particular topological quantum field theory. Here, as also often is the case in literature, we refer to this minimal information as an anyon model. Such models with up to four distinct anyonic quasiparticles have been systematically classified [76]. We refer the interested reader also to the appendices of [40] for an accessible introduction to the diagrammatic notation often used when talking about anyons.

In this section we first give the general structure of anyon models and then illustrate it with two examples most relevant to quantum computation: Fibonacci anyons (universal for quantum computation, but up to now only a theoretical construction) and Ising anyons (not universal for quantum computation, but can be experimentally realized).

\subsection{Fusion channels - Decoherence-free subspaces}

To define an anyon model, one first specifies how many distinct anyons there are. For completeness, this list must include a trivial label, 1 , corresponding to the vacuum with no anyons. The anyon model is then spanned by the particles

$$
M=\{1, a, b, c, \ldots\} .
$$

The labels $a, b, c, \ldots$ can be viewed as topological charges carried by each anyon. As charges they must satisfy conservation rules. For anyons these are known as fusion rules, that take the form

$$
a \times b=\sum_{c \in M} N_{a b}^{c} c
$$

where the fusion coefficients $N_{a b}^{c}=0,1, \ldots$ are non-negative integers describing the possible topological charges (fusion channels) a composite particle of $a$ and $b$ can carry ( $a$ and $b$ are fused). In general $N_{a b}^{c}$ can be any non-negative integer, but for most physical models $N_{a b}^{c}=0,1$. If $N_{a b}^{c}=0$, then fusing $a$ with $b$ can not yield $c$. If for all $a, b \in M$ there is only one $N_{a b}^{c}$ that is different from zero, then the fusion outcome of each pair of particles is unique and the model is Abelian. If for some pair of anyons there are two or more fusion coefficients that satisfy $N_{a b}^{c} \neq 0$, then the model is called non-Abelian. The latter implies that the fusion of $a$ and $b$ can result in several different anyons, i.e. there is degree of freedom associated with the fusion channel. Furthermore, to conserve total topological charge every particle $a$ must have an anti-particle $b$, in the sense that $N_{a b}^{1}=1$ for some $b$. For instance, a fusion rule of the form $a \times a=1+b$, that we encounter below, means that $a$ is a non-Abelian anyon, as it has two fusion outcomes, and that it is its own anti-particle, as one of the fusion outcomes is the vacuum.

The key difference between Abelian and non-Abelian anyons is that the fusion channel degree of freedom enables to define a non-trivial Hilbert space of states spanned by different possible fusion outcomes. That is, if $a$ and $b$ can fuse to several $c \in M$, we can define orthogonal states $|a b ; c\rangle$ that satisfy

$$
\langle a b ; c \mid a b ; d\rangle=\delta_{c d} .
$$

If there are $N$ distinct fusion channels in the presence of a pair of particles, the system exhibits $N$ fold degeneracy spanned by these states. We refer to this non-local space shared by the non-Abelian anyons, regardless of where they are located, as the fusion space. Under the assumption that all microscopics of the system giving rise to the anyons are decoupled from the low-energy physics, the states in the fusion space are perfectly degenerate. As it is a collective non-local property of the anyons, no local perturbation can act on it and it is hence a decoherence-free subspace. As such it is an ideal place to non-locally encode quantum information. We stress that the fusion space arises from the distinct ways anyons can be fused over how they are fused. If two anyons are actually fused and 
the outcome of the fusion is detected, this would correspond to performing a projective measurement in the fusion space.

When there are more than two particles in the system, the anyons can be fused in various different orders and the outcomes of the fusion of different anyons in general depend on the order of the fusion. Nevertheless, consistency conditions need to be satisfied. For fusion rules these take the form of so called pentagon equations, that constrain how fusion outcomes for different fusion orders are related [40]. In the fusion space of many non-Abelian anyons, a basis is given by a fusion diagram of a fixed fusion order spanned by all possible fusion outcomes. Choosing a different fusion order is equivalent to a change of basis. Like the familiar Hadamard gate that relates the $Z$ - and $X$-bases of a qubit, for every anyon model there exists a set of the fusion matrices obtained from the pentagon equations, the $F$-matrices as they are often called, that relate a state in one basis to states in other bases.

Consider a case where three anyons $a, b$ and $c$ are constrained always to fuse to $d$, but there are several intermediate fusion outcomes allowed. There are then two possible fusion diagrams corresponding to distinct bases. Either one fuses first $a$ and $b$ to give $e$, in which case the basis states are labeled by $e$ and denoted by $|(a b) c ; e c ; d\rangle$, or one fuses first $b$ and $c$ to give $f$, in which case the corresponding basis states are $|a(b c) ; a f ; d\rangle$. These two choices of basis must be related by a unitary matrix $F_{a b c}^{d}$ as

$$
|(a b) c ; e c ; d\rangle=\sum_{f}\left(F_{a b c}^{d}\right)_{e f}|a(b c) ; a f ; d\rangle
$$

where $\left(F_{a b c}^{d}\right)_{e f}$ are the matrix elements of $F_{a b c}^{d}$, $f$ is summed over all the anyons that $b$ and $c$ can fuse to. The states and the action of the $F$-matrices are most conveniently expressed in terms of fusion diagrams, as illustrated in Figure 3. Such diagrams also vividly capture the topological nature of such processes - two diagrams that can be continuously deformed into each other (i.e. no cutting or crossing of the world lines) correspond to the same state of the system.

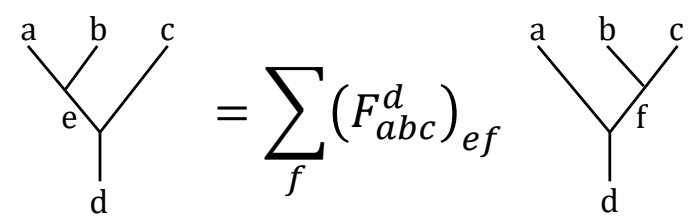

Figure 3: Fusion diagrams as a basis and $F$-matrices. A basis in the fusion space is chosen by choosing an order in which the anyons are to be fused. In the case of three anyons $a, b$ and $c$ that are constrained to fuse to $d$, there are only two options: Either they are fused pairwise from left to right $(|(a b) c ; e c ; d\rangle)$ or from right to left $(|a(b c) ; a f ; d\rangle)$. These two bases are related by the unitary matrix $F_{a b c}^{d}$ according to (7). The state in one basis is in general a superposition of the basis states in the other basis.

\subsection{Statistical quantum evolutions}

The fusion Hilbert space is the collective non-local property of the anyons. To evolve a quantum state in this space, the statistical properties of anyons are employed. In particular, anyons are exchanged, or braided as the process is often called due to their worldlines forming braids. Transporting the anyons along paths that do not enclose other anyons has no effect. For Abelian anyons the fusion space is one dimensional and the only possible evolution is given by a complex phase factor. This phase depends on the type of anyons and whether they are exchanged clockwise or anti-clockwise, but not on the order of the anyon exchanges. In other words, the exchange operator describing exchanging anyons 
$a$ and $b$ in a clockwise fashion is given by $R_{a b}=e^{i \theta_{a b}}$. This contrasts with non-Abelian anyons for which the resulting statistical phase not only depends on the types of anyons, but also on their fusion channel $c$, i.e. the exchange is described by the operator $R_{a b}^{c}=e^{i \theta_{a b}^{c}}$. Thus when there is fusion space degeneracy associated with different fusion channels, braiding assigns different phases to different fusion channels and depends not only on the orientation of the exchanges but also on their order.

Given the $F$-matrices obtained from the pentagon equations, the possible statistics described by the exchange operators $R_{a b}^{c}$ compatible with them can be obtained by solving another set of consistency equations known as hexagon equations [40]. The exchange operators, or the $R$-matrices as they often called, may have several solutions corresponding to the same fusion rules with each solution defining a different anyon model. Consider again the case where anyons $a, b$ and $c$ are constrained to fuse to $d$, as in $(7)$. Then in the basis $|(a b) c ; e c ; d\rangle$ a clockwise exchange of $a$ and $b$ implements the unitary

$$
|(b a) c ; e c ; d\rangle=\sum_{f} R_{a b}^{f} \delta_{e, f}|(a b) c ; e c ; d\rangle,
$$

where $f$ spans all possible fusion outcomes of $a$ and $b$. Thus for a generic state in this basis, a clockwise exchange is represented by a diagonal unitary matrix $R$ with entries $R_{a b}^{f}$. Were the anyons exchanged counter-clockwise, the evolution would be described by $R^{\dagger}$. To write down the effect of exchanging $b$ and $c$ clockwise in the same basis, one first applies the $F_{a b c}^{d}$ to change the basis to $|a(b c) ; a f ; d\rangle$, then applies $R$, as defined above, and then returns to the original basis with $\left(F_{a b c}^{d}\right)^{-1}$, which is given by the unitary evolution

$$
|(a c) b ; e c ; d\rangle=\left(F_{a b c}^{d}\right)^{-1} R\left(F_{a b c}^{d}\right)|(a b) c ; e c ; d\rangle .
$$

All unitary evolutions due to distinct exchanges of anyons can be constructed in similar fashion and they are always given by some combination of the $F$ - and $R$-matrices. Again, these exchange operations are conveniently expressed diagrammatically, as illustrated in Figure 4.
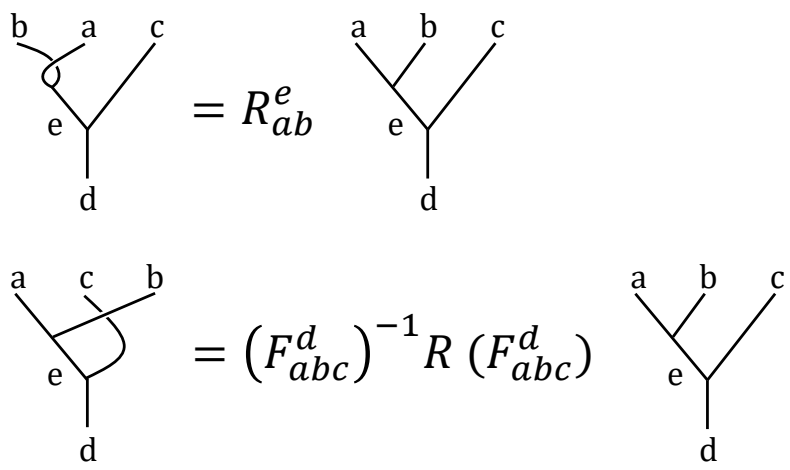

Figure 4: Exchanging anyons in different bases and the $R$-matrices. When two anyons $a$ and $b$ are exchanged in a basis where they are fused first $(|(a b) c ; e c ; d\rangle)$, the $R$-matrix acts as a diagonal matrix that assigns a phase factor $R_{a b}^{e}=e^{i \theta_{a b}^{e}}$ depending on their fusion channel $e$. When $b$ and $c$ are exchanged, the action in the basis where $a$ and $b$ are fused first is obtained by first moving to the basis they are used first $(|a(b c) ; a f ; d\rangle)$ by applying the unitary $F_{a b c}^{d}$, then applying the diagonal $R$-matrix that assigns different phase factors to different fusion channels of $b$ and $c$ and finally returning to the original basis by applying $\left(F_{a b c}^{d}\right)^{-1}$.

Summarizing, an anyon model is most compactly specified by: (i) The fusion coefficients $N_{a b}^{c}$ that describe how many distinct anyons there are and how the anyons fuse, (ii) the $F$-matrices that 
describe the structure of the fusion space and (iii) the $R$-matrices that describe the mutual statistics of the anyons. Regardless of the microscopics that give rise to anyons in a given system, with this minimal set of data all possible states of the fusion space for arbitrary number of anyons and all the possible evolutions can be constructed. As this notation gets rather cumbersome, we illustrate it with two examples that are most relevant to topological quantum computation.

\subsection{Example 1: Fibonacci anyons}

Based on their anyon model structure, Fibonacci anyons are the simplest non-Abelian anyons. There is only one anyon $\tau$ that satisfies the fusion rule

$$
\tau \times \tau=1+\tau
$$

In other words, $\tau$ is its own anti-particle, but two $\tau$ anyons can also behave like a single $\tau$ anyon. To encode a two-dimensional Hilbert space we need three $\tau$ particles that are constrained to fuse to a single $\tau$ particle according to

$$
\tau \times \tau \times \tau=1+2 \tau,
$$

where we have used the associativity of the fusion rules $(10)$. A basis in this two-dimensional fusion subspace can given by the states $|(\tau \tau) \tau ; 1 \tau ; \tau\rangle$ and $|(\tau \tau) \tau ; \tau \tau ; \tau\rangle$. Since both these states have the same $\tau$-parity, they can be superposed and hence a qubit can be encoded in three $\tau$ anyons. For the Fibonacci fusion rules the $F$-matrix and the $R$-matrix are unique and given by

$$
F=F_{\tau \tau \tau}^{\tau}=\left(\begin{array}{cc}
\phi^{-1} & \phi^{-1 / 2} \\
\phi^{-1 / 2} & -\phi^{-1}
\end{array}\right), \quad R=\left(\begin{array}{cc}
R_{\tau \tau}^{1} & 0 \\
0 & R_{\tau \tau}^{\tau}
\end{array}\right)=\left(\begin{array}{cc}
e^{\frac{4 \pi i}{5}} & 0 \\
0 & e^{\frac{-3 \pi i}{5}}
\end{array}\right)
$$

where $\phi=(1+\sqrt{5}) / 2$ is the Golden Ratio (hence the name Fibonacci anyons). For a detailed discussion on Fibonacci anyons, we refer to [77].

This seeming simplicity of the Fibonacci anyons hides something remarkable though. Namely, arbitrary unitaries can be implemented by braiding the Fibonacci anyons and hence the model is universal for quantum computation [31]! Even if $R^{10}$ equals the identity matrix, the braid group generated by $R$ and $F^{-1} R F$ is dense in $S U(2)$ in the sense that an arbitrary unitaries can be approximated to arbitrary accuracy by only braiding the anyons. Approximating even the simplest gates is not simple though with even the NOT-gate requiring thousands of braiding operations in very specific order [78, 79]. Still, the fact that braiding can be employed to generate in principle arbitrary unitaries, as opposed to most other anyon models, makes Fibonacci anyons the Holy Grail for quantum computation.

Unfortunately, the relative simplicity of the Fibonacci anyons themselves does not imply the simplicity of the microscopic physics that could give rise to these anyons. Quite the contrary. While novel elaborate schemes to realize them have been proposed in coupled domain wall arrays of Abelian FQH states [80], the most plausible candidate is still the Read-Rezayi state that has been proposed to describe the filling fraction $v=12 / 5 \mathrm{FQH}$ state [29]. As this state is very fragile, it remains unclear whether it can ever be realized in a laboratory. Hence, much research has focused on states hosting simpler non-Abelian anyons that might not be universal, but which still enable to test and develop topological quantum technologies. In this regard Ising anyons are of particular interest. 


\subsection{Example 2: Ising anyons}

The Ising anyon model consists of two non-trivial particles $\psi$ (fermion) and $\sigma$ (anyon) satisfying the fusion rules

$$
\begin{gathered}
1 \times 1=1, \quad 1 \times \psi=\psi, \quad 1 \times \sigma=\sigma \\
\psi \times \psi=1, \quad \psi \times \sigma=\sigma, \\
\sigma \times \sigma=1+\psi
\end{gathered}
$$

The fusion rule $\psi \times \psi=1$ implies that when brought together two fermions behave like there is no particle, while $\psi \times \sigma=\sigma$ implies that $\psi$ with a $\sigma$ is indistinguishable from a single $\sigma$. The non-Abelian nature of the $\sigma$ anyons is encoded in the last fusion rule which says that two of them can behave either as the vacuum or as a fermion. Physically, the fusion rules can be understood, for instance, in the context of a topological $p$-wave superconductor [47]. There, the vacuum 1 is a condensate of Cooper pairs. The fermions $\psi$ are Bogoliubov quasiparticles that can pair into a Cooper pair and thus vanish into the vacuum. The $\sigma$ anyons, on the other hand, correspond to vortices that bind localised Majorana modes. As we will explain below, a Majorana mode corresponds to a "half" of a complex fermion mode. A pair of such vortices carries thus a single non-local fermion mode, the $\psi$ particle, that can be either unoccupied (fusion channel $\sigma \times \sigma \rightarrow 1$ ) or occupied (fusion channel $\sigma \times \sigma \rightarrow \psi)$.

The non-Abelian fusion rule for the $\sigma$ anyons implies that there is a two dimensional Hilbert space associated with a pair of them. The basis in this Hilbert space can be associated with the two fusion channels and denoted by $\{|\sigma \sigma ; 1\rangle,|\sigma \sigma ; \psi\rangle\}$. However, as these two states belong to different topological charge sectors, they can not be superposed. In order to have a non-trivial fusion space in the same charge sector, one needs to consider at least three $\sigma$ particles that can fuse to a single $\sigma$ in two distinct ways. The basis in this two-dimensional fusion space can be given by the states

$$
\{|(\sigma \sigma) \sigma ; 1 \sigma ; \sigma\rangle,|(\sigma \sigma) \sigma ; \psi \sigma ; \sigma\rangle\}
$$

that correspond to the two left-most $\sigma$ anyons fusing into either 1 or $\psi$. The $F$-matrix to change the basis to fusing from right to left is given by

$$
F=F_{\sigma \sigma \sigma}^{\sigma}=\frac{1}{\sqrt{2}}\left(\begin{array}{cc}
1 & 1 \\
1 & -1
\end{array}\right) .
$$

Since it creates equal weight superpositions, it means that if the fusion outcome is unique in one basis, in the other basis it is completely random. Thus the different fusion orders of Ising anyons correspond to different bases exactly as the basis for a qubit could be chosen along the $z$-axis or along $x$-axis. Unlike Fibonacci anyons, the fusion space of Ising anyons has a natural tensor product structure. The dimension of the Hilbert space doubles for every added $\sigma$ pair and hence for $2 N$ anyons the dimension of the fusion Hilbert space in a fixed parity sector is given by $2^{N-1}$.

In general, the $R$-matrices for the fusion rules (13) are not unique, but admit eight distinct solutions [40]. However, these differ only by the statistical phase $R_{\sigma \sigma}^{\psi}$. For the solution corresponding to Ising anyons, the $R$-matrix in the basis 13 for the clockwise exchange of the two left-most $\sigma$ anyons is given by

$$
R=\left(\begin{array}{cc}
R_{\sigma \sigma}^{1} & 0 \\
0 & R_{\sigma \sigma}^{\psi}
\end{array}\right)=e^{-i \frac{\pi}{8}}\left(\begin{array}{cc}
1 & 0 \\
0 & e^{i \frac{\pi}{2}}
\end{array}\right) .
$$

We immediately see that if we encoded a qubit in the fusion space associated with three $\sigma$ particles, $R^{2}$ would implement the logical phase-gate up to an overall phase factor. If the two right-most anyons 
were instead exchanged twice, the evolution in the basis (13) would be described by

$$
F^{-1} R^{2} F=e^{-i \pi 4}\left(\begin{array}{cc}
0 & 1 \\
1 & 0
\end{array}\right) \text {. }
$$

In other words, braiding them changes the fusion channel of the two left most ones between 1 and $\psi$. If the three $\sigma$ 's were employed to encode a qubit, this braid would have implemented a logical $U_{N O T}$-gate.

The limitation of the operations that can be performed is obvious from the braiding of three $\sigma$ anyons. Since one can only implement logical phase and $U_{N O T}$-gates on a single qubit, Ising anyons can only implement the Clifford group by braiding [81, 82]. This means that Ising anyons, while being non-Abelian, are not universal for quantum computation by braiding. To overcome this shortcoming, non-topological schemes have been devised to promote their computational power to universality. While the need for such non-topological operations makes the system more susceptible to errors, Ising anyons are still the best candidates to experimentally test the principles of topological quantum computation due to their realization in superconducting nanowire heterostructures [58, 59, 60, 61, 62]. In the Section 3 we describe in more detail how a topological quantum computer would in general be operated and then Section 5 illustrate these steps in the context of the experimentally relevant Majorana wires.

\section{Quantum computation with anyons}

While discussing non-Abelian anyons and the non-local fusion space associated with them, we have already hinted how this space could be used to encode and process quantum information in an inherently fault-resilient manner. In the ideal conditions of zero temperature and infinite anyon separation, states in the fusion space have three very attractive properties:

(i) All the states are perfectly degenerate.

(ii) They are indistinguishable by local operations.

(iii) They can be coherently evolved by braiding anyons.

If this space of states is used as the logical space of a quantum computer, property (i) implies that the encoded information is free of dynamical dephasing, while property (ii) means that it is also protected against any uncorrelated local perturbations. Property (iii) means that errors could only occur under unlikely non-local perturbations to the Hamiltonian that would create virtual anyons and propagate them around the encoding ones. However, braiding of the anyons can still be carried out robustly by the operator of the computer to execute desired quantum gates. Furthermore, property (iii) implies that all the quantum gates are virtually free from control errors since they depend only on the topological characteristics of the braiding evolutions given by the $F$ - and $R$-matrices.

Together these properties mean that quantum computation with anyons would heavily suppress errors already at the level of the hardware, with little need for resource intensive quantum error correction. Of course, in the real world these ideal conditions are never met and some decoherence of the encoded information always takes place. Still, the topological encoding and processing of quantum information provides in principle unparalleled protection over non-topological schemes. For the time being we forget about the nasty reality and focus on outlining the steps to operate a topological quantum computer with Ising anyons as our case study. Generic error sources present in laboratory are discussed at the end of the section. 


\subsection{Initialization of a topological quantum computer}

To initialize a quantum computer, one needs first to identify the computational space of $n$ qubits. In topological computer this means creating some number of anyons from the vacuum and fixing their positions. The system then exhibits a fusion space manifesting as ground state degeneracy that is identified as the computational space. Depending on the anyons in question, the full fusion space may not admit a tensor product structure (such as the Fibonacci anyons), but one can always identify subspaces corresponding to the fusion channels of subsets of anyons that can serve as qubits. To illustrate the steps of operating a topological quantum computer, we focus on the simpler Ising anyons whose fusion space does exhibit natural tensor product structure.

For $2 N$ Ising anyons the fusion space dimension increases exponentially as $D=2^{N}$. To initialize the system, we assume that $\sigma$ anyons are created pairwise from the vacuum with no other anyons present. This means that every pair is initialized in the $\sigma \times \sigma \rightarrow 1$ fusion channel and hence the system globally belongs also to the vacuum sector. Due to this constraint, in this sector the fusion space has the dimension $D=2^{N-1}$. Thus four $\sigma$ anyons encodes a qubit, six $\sigma$ 's encodes two qubits, and so on. Let us focus on a system of six $\sigma$ anyons that enables to demonstrate all the basic operations. It is convenient to choose a computational basis as the pairwise fusion basis

$$
\begin{aligned}
|00\rangle & =|\sigma \sigma ; 1\rangle|\sigma \sigma ; 1\rangle|\sigma \sigma ; 1\rangle, \\
|10\rangle & =|\sigma \sigma ; \psi\rangle|\sigma \sigma ; \psi\rangle|\sigma \sigma ; 1\rangle, \\
|01\rangle & =|\sigma \sigma ; 1\rangle|\sigma \sigma ; \psi\rangle|\sigma \sigma ; \psi\rangle, \\
|11\rangle & =|\sigma \sigma ; \psi\rangle|\sigma \sigma ; 1\rangle|\sigma \sigma ; \psi\rangle,
\end{aligned}
$$

where the three kets refer to the fusion channels of the three $\sigma$ pairs, as illustrated in Figure 5. When the anyon pairs are created from the vacuum, the topological quantum computer is initialized in the state $|00\rangle$. The other basis states involve an even number of intermediate $\psi$ channels, which according to $\psi \times \psi=1$ is consistent with the constraint that the fusion of all $\sigma$ anyons must always yield the vacuum.

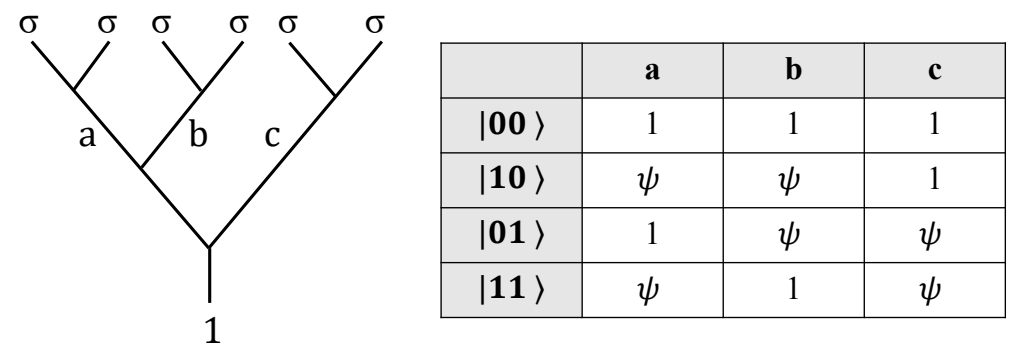

Figure 5: The fusion diagram for six Ising anyons for a pairwise basis restricted to the global vacuum sector. Due to the fusion rules $\sigma \times \sigma=1+\psi$ and $\sigma \times \psi=\sigma$, the fusion diagram contains four distinct fusion channels that are consistent with the constraint that the fusion of all the six $\sigma$ anyons must give the vacuum 1. The table shows the identification of the fusion channels with the computational basis of two qubit.

\subsection{Quantum gates - Braiding anyons}

To perform a computation in the fusion space is equivalent to specifying a braid - a sequence of exchanges of the anyons that corresponds to the desired sequence of logical gates. For Ising anyons, 
the natural gate set to implement consists of Clifford operations on single qubits, i.e. the $X-, Z$ - and Hadamard $U_{H}$-gates and the two-qubit controlled phase gate $U_{C Z}$.

The single qubit gates follow directly from the $F$ - and $R$-matrices of Ising anyons, (15) and (16). The first says that under two exchanges the state acquires a -1 phase whenever the exchanged $\sigma$ pair fuses to a $\psi$, while the latter says that exchanging twice two $\sigma$ anyons from different pairs simultaneously changes the fusion channel of both pairs between 1 and $\psi$. In the two-qubit computational basis (17), the elementary single qubit operations, up to an overall phase, are thus given by

$$
\begin{array}{ll}
X_{1}=R_{23}^{2}=F^{-1} R^{2} F \otimes \mathbb{I}, & Z_{1}=R_{12}^{2}=R^{2} \otimes \mathbb{I}, \\
X_{2}=R_{45}^{2}=\mathbb{I} \otimes F^{-1} R^{2} F, & Z_{2}=R_{56}^{2}=\mathbb{I} \otimes R^{2}
\end{array}
$$

where $R_{i j}$ is the clockwise exchange operator acting on anyons $i$ and $j$. The corresponding braiding diagrams are illustrated in Figure 6. Similarly, the Hadamard gates $U_{H}$ can be implemented by single exchanges. One can easily verify that $F^{-1} R F$ creates superpositions of fusion channels, but with distinct phase factors assigned to different fusion channels. These phase factors can be cancelled by appending it further exchanges. A little algebra shows that up to an overall phase, the Hadamard gates on the two qubits are given by

$$
\begin{aligned}
& U_{H, 1}=R_{12} R_{23} R_{12}=R F^{-1} R F R \otimes \mathbb{I}, \\
& U_{H, 2}=R_{56} R_{45} R_{56}=\mathbb{I} \otimes R F^{-1} R F R,
\end{aligned}
$$

as illustrated in Figure 6. Hence by braiding only one can implement all the single qubit operations in the Clifford group.

To implement two-qubit operations, one needs to perform braids outside this group of operations. The natural two-qubit gate to implement with Ising anyons is the controlled- $Z$ gate, that is given by the braid

$$
U_{C Z}=R_{12}^{-1} R_{34} R_{56}^{-1}
$$

Since the three exchanges act on separate pairs of $\sigma$ anyons, the action of such braid can be deduced piecewise in the computational basis (17). First, $R_{34}$ gives rise to unitary that maps $|01\rangle,|10\rangle \rightarrow$ $e^{i \frac{\pi}{2}}|01\rangle, e^{i \frac{\pi}{2}}|10\rangle$ by assigning the phase factor $e^{i \frac{\pi}{2}}$ whenever the middle pair is in the $\psi$ fusion channel. Likewise, $R_{12}^{-1}$ acts on the left pair and maps $|10\rangle,|11\rangle \rightarrow e^{-i \frac{\pi}{2}}|10\rangle, e^{-i \frac{\pi}{2}}|11\rangle$, while $R_{56}^{-1}$ acts on the right pair and maps $|01\rangle,|11\rangle \rightarrow e^{-i \frac{\pi}{2}}|01\rangle, e^{-i \frac{\pi}{2}}|11\rangle$. Hence, the joint effect is to map only $|11\rangle \rightarrow-|11\rangle$, which is precisely the controlled- $Z$ gate between the two qubits.

Unfortunately, this is the best one can do with Ising anyons. A two-qubit gate together with Clifford group operations does not form a universal gate set for quantum computation. For that one requires an additional non-Clifford gate, such as the $\frac{\pi}{8}$-phase gate [83, 84]. In principle, it can be implemented non-topologically by bringing two anyons nearby. An interaction between the anyons will then lift the degeneracy of the fusion channels by $\Delta E$ and they will dephase in time according to

$$
U=\left(\begin{array}{cc}
1 & 0 \\
0 & e^{i \Delta E t}
\end{array}\right)
$$

Assuming that bringing the anyons nearby and separating them again can be controlled precisely such that $\Delta E t=\pi / 4$, than one would have implemented the desired $\frac{\pi}{8}$-phase gate, albeit in a nontopologically protected fashion. While this is likely to be challenging in the laboratory, more elaborate schemes have been proposed to implement this gate in a robust manner [85, 86, 87]. 


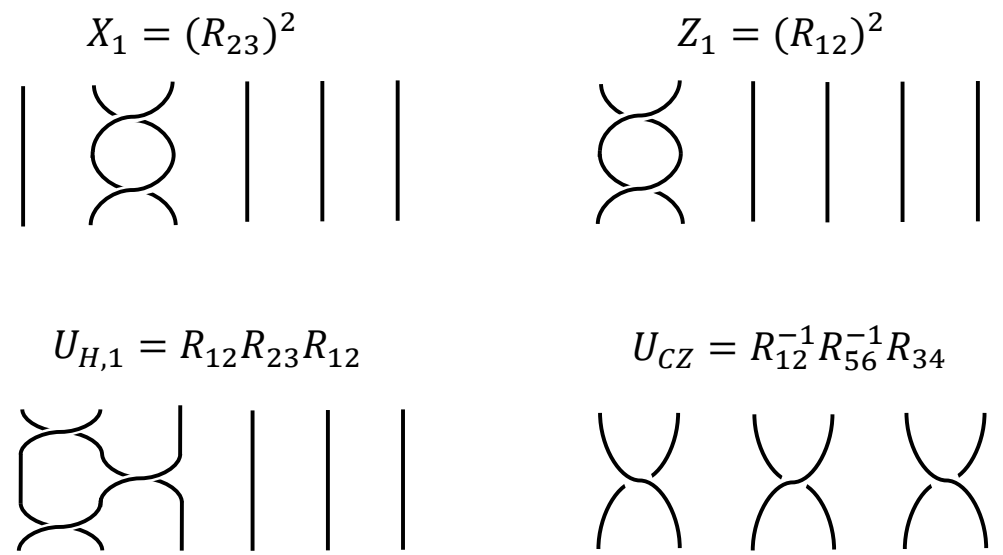

Figure 6: The elementary braids corresponding to the $X-, Z$ - and Hadamard $U_{H}$-gates on the first qubit as well as the controlled- $Z$ gate $U_{C Z}$. The single qubit operations on the second qubit are given by (18) and (19).

\subsection{Measurements - Fusing anyons}

The final step of a computation is the read-out. As the computational basis states (17) correspond to distinct patters of pairwise fusion outcomes, the projective measurements are performed by bringing the anyons pairwise physically together and detecting the fusion outcomes. How this is done in practice depends on the anyons in question and on the microscopic physics that give rise to them. For the Ising anyons there is in principle a straightforward way to do this. A pairwise fusion can result only either in the vacuum (nothing remains) or the $\psi$ anyon (a massive particle remains). This distinction in the change of energy of the system is in principle detectable and serves as a method to perform measurements in the computational basis.

In our example of two qubits encoded in six $\sigma$ anyons illustrated in Figure 5, a projective $Z$-basis measurement of the first qubit corresponds to detecting the fusion outcome of anyons 1 and 2 . If no change in energy is detected, then one has applied the projector $|0\rangle\langle 0| \otimes \mathbb{I}$, while observing a change in energy applies the projector $|1\rangle\langle 1| \otimes \mathbb{I}$. Before the measurement is applied the state of the system could be in a superposition of fusion states. Similarly, an $X$-basis measurements are performed by detecting the fusion outcome of anyons 2 and 3. For measurements on the second qubit the same operations are applied to anyons 5 and 6 for $Z$-basis measurements and to anyons 4 and 5 for $X$-basis measurements. By measuring the fusion outcomes of other anyon pairs, one can apply projectors into joint subspaces of the two qubits. For instance, detecting that anyons 3 and 4 fuse to vacuum projects into the subspace spanned by $|00\rangle$ and $|11\rangle$, while observing $\psi$ projects onto its complement.

In a nutshell, these are the basic steps of operating a topological quantum computer. How they are carried out precisely depends on the microscopics of the system that supports the anyons. In Section 5 we put these ideas in a more concrete setting and consider how a topological quantum computation could in principle be carried in one microscopic system that supports Ising anyons. Before proceeding there, we discuss briefly how a topological quantum computer might fail to deliver the promised protection under realistic conditions. 


\subsection{Possible error sources}

The biggest experimental challenge to topological quantum computation is that anyons, especially of the non-Abelian kind, are hard to come by theoretically, and even more experimentally. Assuming that one would have access to anyons, there are still several mechanisms that topological quantum protection might fail despite of its inherent resilience to local non-correlated perturbations.

Spurious anyons: Like with conventional qubits, decoherence in topological quantum computation can arise due to an uncontrolled coupling to a reservoir. In topologically ordered systems the reservoir could appear in two ways. Either there are additional anyons in the system, causing the full fusion space to enlarge beyond the computational space, or there is a reservoir nearby from where quasiparticles can tunnel into and out of the system. Considering that anyons require precise microscopic conditions, it should be relatively easy to take care of the latter by ensuring that there are no accidental topologically ordered states nearby. The first source of a reservoir, however, requires care. As any topological quantum computation is likely to require a macroscopic number of anyons to define a useful computational space (it is estimated that for a robust implementation of quantum algorithms this number ranges from $10^{3}$ Fibonacci anyons to a whopping $10^{9}$ Ising anyons [79]), it is in general hard to keep track of all the anyons in the system. When executing quantum gates, braiding around unaccounted anyons would then rotate the state outside the computational space. If the unaccounted anyons are tightly paired and localized, this will not be a problem as their fusion channel is fixed to the vacuum channel and braiding around both has no effect. If the anyons can propagate freely though, accidental braids around isolated anyons can occur leading to decoherence.

Anyon-anyon interactions: The exact degeneracy of the states corresponding to different fusion channels relies on the anyons being completely non-interacting or far away so their decaying interactions become negligible and thus unable to distinguish the fusion channels by lifting their degeneracy. In the laboratory everything is finite though and when a macroscopic number of anyons is required to operate a topological quantum computer, it is impossible to keep all of them well separated at all times. What far away means for a certain system is given by the coherence length that scales in general as the inverse energy gap $\xi \sim \Delta^{-1}$. As the anyons are quasiparticles, that are collective states of the elementary electrons, they are in practice always exponentially localized with the decay of the wave function controlled by $\xi$. Thus when two anyons with two possible fusion channels are within distance $L$ of each other, virtual tunneling processes between them lift the degeneracy of the fusion channels by $\Delta E \sim e^{-L / \xi}$ [88]. Indeed, this splitting has been calculated in $p$-wave superconductors [89], fractional quantum Hall states [90] and spin liquids [91], and also experimentally observed for Majorana zero modes in superconducting nanowires [62].

Lifting of the fusion channel degeneracy implies several subtleties for topological quantum computers. First, logical states with different energies will dephase with time. While they remain insensitive to local operations, the Hamiltonian of the system will distinguish between them akin to the nontopological implementation of the $\frac{\pi}{8}$-phase gate 21 . Thus topological qubits, like non-topological ones, will decohere with time unless error correction is applied [92]. Second, quantum gates by braiding are no longer necessarily exact. Finite energy splitting between the ground states implies that the evolution must be fast enough at the scale of $\Delta E$ for the states to appear degenerate, while still being slow enough at the scale of the energy gap $\Delta$ not to excite the system [93, 94]. In general this balancing between the two energy scales means that there are small errors in the implemented logical operations. If they accumulate and are not error corrected, they will become a source of decoherence.

Anyon-anyon interactions can also induce topological phase transitions when the anyons form regular arrays [95, 96, 97]. Any scalable architecture for a topological quantum computer would surely employ a systematic arrangement of anyons to keep track of them. As a transition to a different 
topological phase of matter is the ultimate failure of a topological quantum computer, it needs to be avoided at all costs. The microscopic conditions for such anyon-anyon interaction induced phase transitions, particularly those for arrays of localized Majorana modes, have been studied in several works [98, 99, 100, 101, 102, 103].

Finite temperature: In principle the energy gap $\Delta$ of topologically ordered systems protects the encoded information by suppressing thermal fluctuations as $e^{-\frac{\Delta}{k T}}$. Such fluctuations are detrimental to topological quantum computation. In the presence of thermally excited anyons the statistics of anyons as is expected to become ill-defined and braiding no longer corresponds to desired quantum gates. However, in intrinsically topologically ordered states the energy gap might not be enough. Studies on Abelian anyon-based topological quantum memories suggest that any finite temperature, even if much smaller than the gap, can be a problem [104, 105, 106]. Any finite system, and in a laboratory everything is finite, does have critical threshold temperature, but again as extensive amounts of anyons are in general needed, such protection in realistic finite-size systems is unlikely to be sufficient for stability. Promisingly, this may be circumvented if the system possesses some mechanism that suppresses the spontaneous creation of stray anyons [107, 108, 109, 110, 111], but it is unclear whether such schemes are realistic. Experimentally the situation is equally challenging as the energy gaps tend to be small and thus only formidably low temperatures can be tolerated even for short times.

\section{Manifestations of anyons in microscopic many-body systems}

So far we have discussed non-Abelian anyons at an abstract level. In reality, the anyons appear as collective massive quasiparticles in strongly correlated many-body systems. In this section we first discuss how the key properties of anyons, the fusion space and braiding evolutions manifest themselves in general in a microscopic many-body system. Furthermore, we introduce an entanglement signature of anyons - the topological entanglement entropy - that is an important diagnostic tool to identify whether a given state supports them.

\subsection{Ground state degeneracy and Berry phases}

By definition, in a gapped topological state of matter the ground state is separated from the rest of the states in the spectrum by a spectral gap $\Delta$. When a system is placed on a surface of trivial topology without boundaries, such as a sphere, then the ground state is unique. However, when massive anyonic quasiparticle excitations are introduced into the system, such as some number of $\sigma$ anyons, the lowest energy state in their presence exhibits degeneracy $D$ corresponding to the fusion space associated with them, as illustrated in Figure 7. This non-local degenerate manifold of states is in general exponentially degenerate in the anyon separation (the microscopics induced anyon-anyon interactions are negligible) and it is separated from any other states by the spectral gap $\Delta$. This gap protects the quantum information encoded in the fusion space by supressing any unwanted anyonic excitations in the system.

Braiding of anyons results in an evolution in this degenerate manifold when the anyonic quasiparticles are transported adiabatically around each other. Physically this is captured by the non-Abelian Berry phase acquired by the system's wave function under such a process [112, 113, 114]. Let us consider a system of $N$ non-Abelian anyons that give rise to $D$-dimensional fusion space, where $D$ depends on $N$ and on the type of the non-Abelian anyons. The fusion space manifests itself as the $D$ 


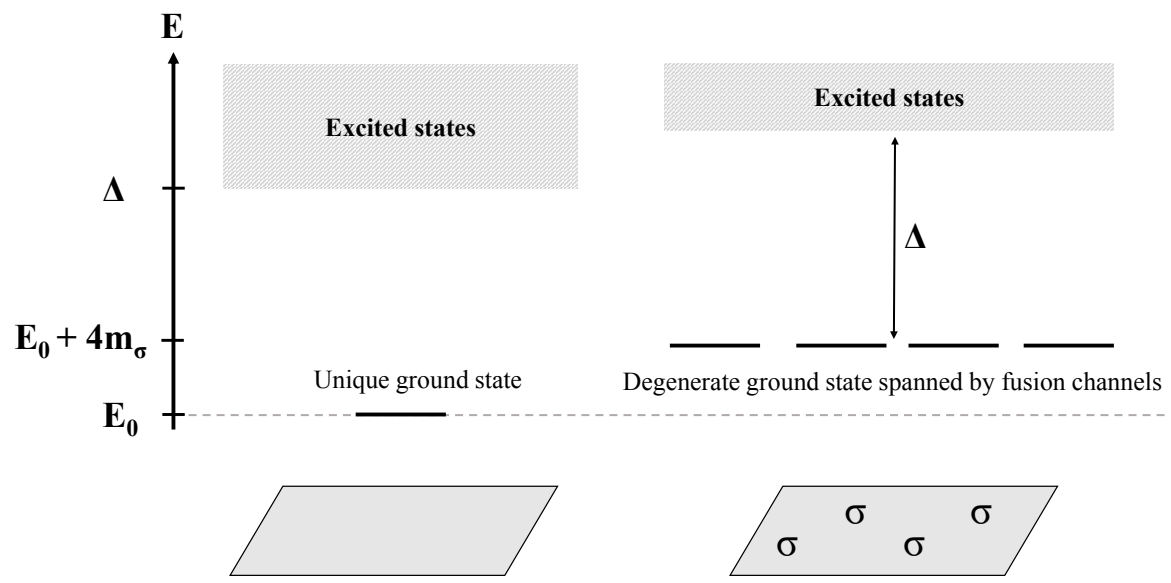

Figure 7: When a topologically ordered state is placed on a surface of trivial topology and no anyons are present, the ground state at some energy $E_{0}$ is unique and separated from excited states by an energy gap $\Delta$. In the presence of non-Abelian anyons the lowest energy state exhibits degeneracy due to the fusion space shared by the anyons (e.g. four-fold degenerate if four $\sigma$ anyons of the Ising model are present with two states belonging to the vacuum sector and two states to the $\psi$-sector). Since anyons are massive excitations, this degenerate manifold appears at some higher energy than the ground state in the absence of anyons (e.g. at energy $E_{0}+4 m_{\sigma}$ when each $\sigma$ anyon has mass $m_{\sigma}$ ), but it is still separated from excited states by the spectral gap $\Delta$.

degenerate many-body ground states given by

$$
\left|\Psi_{n}\left(z_{1}, z_{2}, \ldots, z_{N}\right)\right\rangle, \quad n=1,2, \ldots, D
$$

that depend in general on the anyon coordinates $z_{j}$. Let $\lambda$ be a cyclic path in $z_{j}$ that winds one anyon around another, as illustrated in Figure 8. If one changes the parameters $z_{j}$ slowly in time compared to the energy gap $\Delta$, then the transport is adiabatic and the system evolves only within the degenerate ground state manifold spanned by the states 22). This evolution is in general given by

$$
\left|\Psi_{n}\left(z_{1}, z_{2}, \ldots, z_{N}\right)\right\rangle \rightarrow \sum_{m=1}^{D} \Gamma_{n m}(\lambda)\left|\Psi_{m}\left(z_{1}, z_{2}, \ldots, z_{N}\right)\right\rangle,
$$

where the non-Abelian Berry phase is defined by

$$
\Gamma(\lambda)=\mathbf{P} \exp \oint_{\lambda} \mathbf{A} \cdot d \mathbf{z}
$$

Here $\mathbf{P}$ denotes path ordering and the components of the non-Abelian Berry connection are given by

$$
\left(A^{j}\right)_{m n}=\left\langle\Psi_{m}\left(z_{1}, z_{2}, \ldots, z_{N}\right)\left|\frac{\partial}{\partial z_{j}}\right| \Psi_{n}\left(z_{1}, z_{2}, \ldots, z_{N}\right)\right\rangle .
$$

The geometric phase due to the cyclic evolution in the coordinates $z_{j}$ does not depend on the time it takes to traverse the path $\lambda$ as long as it is long enough for the evolution to be adiabatic. Nor does it depend on the exact shape of the path. The unitary $\Gamma(\lambda)$ is thus topological in nature and must coincide with the respective unitary constructed from $F$ - and $R$-matrices of the corresponding anyons. 


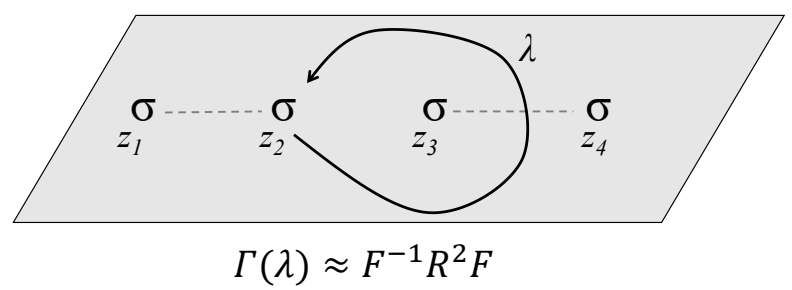

Figure 8: Microscopic braiding as a non-Abelian Berry phase. Consider a system where two pairs of Ising $\sigma$ anyons are created from the vacuum (the pairs are connected by dashed lines) located at positions $z_{1}, \ldots, z_{4}$. Such a system is restricted to the total vacuum sector and exhibits two-fold ground state degeneracy. Transporting the anyon at position $z_{2}$ along any path $\lambda$ that encloses only the anyon at $z_{3}$ gives rise to non-Abelian Berry phase (24). When performed adiabatically, the Berry phase will accurately approximate the braid matrix $F^{-1} R^{2} F$ of the Ising anyons 16 and act on the degenerate ground state manifold shown in Figure 7 .

It has been verified in several microscopically distinct settings that the statistics of the anyons is indeed obtained from the adiabatic evolution of their wave functions. Analytically this has been shown for the Laughlin [115] and Moore-Read states [116] fractional quantum Hall states, as well as $p$-wave superconductors [117] including nanowire heterostructure [118, 119]. These calculations are supported by numerics that have been used to demonstrate non-Abelian statistics in more complex fractional quantum Hall states [120, 90, 121] and in microscopic models such as Kitaev's honeycomb lattice model [93, 122] or bosonic fractional quantum Hall systems [123].

\subsection{Topological degeneracy and entanglement entropy}

We have discussed how the fusion space of anyons manifests itself as the degeneracy of the ground state in the presence of anyonic quasiparticle excitations. Intriguingly, the anyonic excitations of a given topologically ordered system are encoded already in their ground states. This property is due to the long-range correlations present in such states that enable the anyonic nature to be probed without considering excited states in the first place.

A curious consequence of the long-range correlations is that topologically ordered states are sensitive to the topology of the surface they are defined on [21]. If instead of a sphere the state is placed on a surface of non-trivial topology, such as a torus (formally, a genus one surface due to the single hole in it), then the ground state exhibits degeneracy even if no non-Abelian anyons are present. For a state supporting Abelian anyons on genus one surfaces, the degeneracy equals the number of distinct anyons (including the vacuum). For states with non-Abelian anyons and/or Abelian anyons on higher genus surfaces, the degeneracy is most conveniently obtained from the Verlinde formula [124]. Thus by considering the scaling of the degeneracy of the ground state on different genus surfaces, one can obtain information that narrows down, the nature of the anyons a given system supports. The nature of the anyons can not unambiguously identified though, since several topologically ordered models can exhibit the same scaling.

The long-range correlations also have a fingerprint in the entanglement entropy present in the ground state. All gapped states of matter are expected to follow the area-law scaling of entanglement entropy. It has been shown that if a state is topologically ordered, there is a subleading universal constant correction to the area-law [16, 17, 18]. In other words, if the system is partitioned into regions $A$ and $B$, and $|\partial A|$ denotes the length of the boundary between them, then the entanglement 
entropy of a topologically ordered state scales as

$$
S=\alpha|\partial A|-\gamma
$$

where $\alpha$ is a non-universal constant, but $\gamma$ is a universal constant known as topological entanglement entropy. Like the degeneracy scaling with increasing surface genus, this constant is related to the anyon model the state supports, but does not unambiguously identify it. Formally, it is given by the total quantum dimension of the anyon model. To determine the total quantum dimension note that the fusion rules 5 ) can also be written as polynomial equation $d_{a} d_{b}=\sum_{c} N_{a b}^{c} d_{c}$, where $d_{a} \geq 1$ is the quantum dimension of anyon $a$. Clearly, $d_{a}=1$ for Abelian anyons, while $d_{a}>1$ for non-Abelian anyons. The total quantum dimension of the model is defined as $D_{M}=\sqrt{\sum_{a \in M} d_{a}^{2}}$ and in terms of it the topological entanglement entropy is given by $\gamma=\log D_{M}$.

Extracting $\gamma$ by studying the entropy scaling (26) enables thus conveniently to determine whether a given state supports anyons. In particular, it narrows down the possible supported anyon models since, like the topological degeneracy, $D_{M}$ is not a unique characteristic of an anyon model. To unambiguously determine the anyon model, the statistics of the anyons need to be obtained from the ground state as a Berry phase associated with topological twists [125, 126, 127].

We should mention one more characteristic of topological states. If instead of a closed manifold the state is placed on a surface with a boundary, topologically ordered states can exhibit gapless states on their edges [128]. If they do, then their spectrum, which is usually described by a conformal field theory, is in one-to-one correspondence with the possible bulk anyon excitations. This so called bulkboundary correspondence enables to probe the nature of the bulk state by studying the edge spectrum of the ground state. The same information can also be obtained from the entanglement spectrum of the bulk state [129, 32] that has been shown to be in one-to-one with the spectrum of the edge states [130].

\section{Majorana zero modes in a superconducting nanowire}

Finally, we turn to illustrating the principles of topological quantum computation in the context of a microscopic model that supports Ising anyons - Kitaev's toy model for a superconducting nanowire. Strictly speaking it is not topologically ordered in the sense described above since it is 1D model and as such does not exhibit topological degeneracy or topological entanglement entropy. Also, the anyons are not strictly speaking Ising anyons, but Majorana zero modes that are not intrinsic excitations of the state, but appear only on domain walls. Still, for all practical purposes, including the protected fusion space and implementing quantum gates by braiding, they behave as such.

Since the work of Ivanov [20], it has been known that quasiparticles in a two-dimensional system described by localized Majorana modes exhibit the braiding statistics of Ising anyons. Formally, Majorana modes are "half" of a complex fermion mode. By this we mean that if $f$ is a fermion operator satisfying $\left\{f^{\dagger}, f\right\}=1$, one can always write

$$
f_{i}=\frac{1}{2}\left(\gamma_{1}+i \gamma_{2}\right)
$$

where $\gamma_{i}=\gamma_{i}^{\dagger}$ are Hermitian Majorana operators satisfying $\left\{\gamma_{i}, \gamma_{j}\right\}=2 \delta_{i j}$ and $\gamma_{i}^{2}=1$. If two Majorana modes, $\gamma_{1}$ and $\gamma_{2}$, would exist as localised quasiparticles, then the occupation $f^{\dagger} f=0,1$ of the complex fermion shared by them would constitute a non-local degree of freedom. This would precisely correspond to the non-local degree of freedom of two $\sigma$ particles of the Ising anyon model 
13. If the fermionic mode is unoccupied $\left(f^{\dagger} f=0\right)$, then the two Majorana modes would behave like the vacuum, 1 , when brought together, while if it is occupied $\left(f^{\dagger} f=1\right)$, then the fusion of two such quasiparticles would leave behind the fermion $\psi$.

Writing a complex fermion operator as a linear combination of two Majorana operators is a mathematical identity, that by no means implies that Majorana fermions could actually appear on their own. Ettore Majorana suggested their presence in 1937 as 3D fermionic particles that are their own antiparticles. Their restriction to $2 \mathrm{D}$ brings about a non-Abelian anyonic behaviour to the wave function that describes them, which is not present in their 3D counterpart. Such realizations can be met in condensed matter systems. Pioneering work on $p$-wave superconductors [47] suggested though that such exotic 2D superconductors might host Majorana modes localized at vortices. Building on this work, Kitaev proposed a simplified 1D toy model where Majorana modes could appear at the ends of a superconducting wire [55]. While not having a clear experimental realization at the time, this model provided the invaluable insight that domain walls can also host Majorana modes. It took 10 years to discover that Kitaev's simple model could be realized by depositing a spin-orbit coupled semiconducting nanowire on top of a normal $s$-wave superconductor [56, 57]. Remarkably, a few years later experimentalists confirmed this prediction [58, 59, 60, 61]. As discussed in Section 1.2, numerous microscopically distinct proposals for realizing Majorana modes have since been put forward. Regardless of the microscopics though, the low energy physics in all of them can always be cast in the form of the original toy model [55].

\subsection{Kitaev's toy model for a topological nanowire}

Consider a system of spinless superconducting fermions on a 1D lattice of length $L$ described by the Hamiltonian

$$
H=\sum_{j=1}^{L}\left[-t\left(f_{j}^{\dagger} f_{j+1}+f_{j+1}^{\dagger} f_{j}\right)-\mu\left(f_{j}^{\dagger} f_{j}-\frac{1}{2}\right)+\left(\Delta_{p} f_{j} f_{j+1}+\Delta_{p}^{*} f_{j+1}^{\dagger} f_{j}^{\dagger}\right)\right] .
$$

Here $t$ is the tunnelling amplitude, $\mu$ is the chemical potential and $\Delta_{p}=\left|\Delta_{p}\right| e^{i \theta}$ is the superconducting pairing potential. Following [55], this Hamiltonian for $L$ complex fermions can be written in terms of $2 L$ Majorana operators. Absorbing the superconducting phase $\theta$ into the definition (27) by writing $f_{j}=e^{-i \theta / 2}\left(\gamma_{2 j-1}+i \gamma_{2 j}\right) / 2$, the Hamiltonian takes the form

$$
H=\frac{i}{2} \sum_{j=1}^{L}\left[-\mu \gamma_{2 j-1} \gamma_{2 j}+\left(t+\left|\Delta_{p}\right|\right) \gamma_{2 j} \gamma_{2 j+1}+\left(-t+\left|\Delta_{p}\right|\right) \gamma_{2 j-1} \gamma_{2 j+2}\right] .
$$

There are two special limits in which the ground state can be obtained immediately. When the chemical potential term dominates, we can set $\left|\Delta_{p}\right|=t=0$. The Hamiltonian becomes

$$
H=-\frac{i}{2} \sum_{j=1}^{L} \mu \gamma_{2 j-1} \gamma_{2 j}=-\mu \sum_{j=1}^{L}\left(f_{j}^{\dagger} f_{j}-\frac{1}{2}\right), \quad \mu \gg t,\left|\Delta_{p}\right|
$$

which means that the ground state is given by having a fermion $\left(f_{j}^{\dagger} f_{j}=1\right)$ on every site, as illustrated in Figure 9. This state is a product state of fermion modes localized on physical sites and hence topologically trivial.

The other limit is to have the kinetic term comparable to the pairing potential and dominating over the chemical potential. Setting $t=\left|\Delta_{p}\right|$ and $\mu=0$, we obtain the Hamiltonian

$$
H=i t \sum_{j=1}^{L} \gamma_{2 j} \gamma_{2 j+1}=2 t \sum_{j=1}^{L-1}\left(\tilde{f}_{j}^{\dagger} \tilde{f}_{j}-\frac{1}{2}\right), \quad t=\left|\Delta_{p}\right| \gg \mu
$$


where we have defined a new set of fermionic operators by combining the Majoranas as $\tilde{f}_{j}=e^{-i \theta / 2}\left(\gamma_{2 j}+\right.$ $\left.i \gamma_{2 j+1}\right) / 2$ from different physical sites. As illustrated in Figure 9, the Majorana operators $\gamma_{1}$ and $\gamma_{2 L}$ at the edges completely decouple from the Hamiltonian that now describes interactions only between $L-1$ complex fermions. The missing fermion can be described by the operator $d=e^{-i \theta / 2}\left(\gamma_{1}+i \gamma_{2 L}\right) / 2$ that is delocalised between the two ends of the wire. Since $\left[d^{\dagger} d, H\right]=0$, the ground state of this idealized system is two-fold degenerate with the two ground states parametrized by the population of the $d$-mode. Formally this mode can be added to Hamiltonian (31) by assigning zero energy to it. For this reason the edge Majorana modes appearing in topological wires are also called Majorana zero modes. As opposed to the trivial phase in the $t=\left|\Delta_{p}\right|=0$ limit, we call this phase a topological phase since the ground state degeneracy arises from fractionalized Majorana edge states (for periodic boundary conditions the ground state is unique). More formally, the ground state is characterised by a topological invariant [55].

Of course, the coupling regimes $t=\left|\Delta_{p}\right|=0$ or $\mu=0$ are only idealized limiting cases. Were the model realised in the topological phase, one would hope to achieve a parameter regime where $t \approx\left|\Delta_{p}\right|>\mu$. This is fine as the system remains in the topological phase that supports localised Majorana modes at wire ends as long as $t>|\mu|$ (the model is exactly solvable with a Fourier transform followed by a Bogoliubov transformation [55]). The consequence of being away from the extreme $\mu=0$ point is that the Majorana modes will be exponentially localised at the wire ends rather than being positioned on a single site. The operators describing them at the left $(\mathrm{L})$ and right $(\mathrm{R})$ ends of the wire take the general form

$$
\Gamma_{L / R}=\sum_{j=1}^{2 L} \alpha_{j}^{L / R} \gamma_{j} .
$$

The normalised amplitudes decay as $\left|\alpha_{j}^{L}\right| \propto e^{-j / \xi}$ and $\left|\alpha_{j}^{R}\right| \propto e^{-(2 L-j) / \xi}$, where $\xi \propto \Delta^{-1}$ is the coherence length and $\Delta$ the spectral gap that separates the degenerate ground states from the rest of the spectrum. This means that for finite wires of length $L$, the wave functions of the two Majoranas will in general overlap, which in turn results in a finite energy splitting $\Delta E \propto e^{-L / \xi}$ between the two ground states. This splitting is negligible for very long wires. Nevertheless, it serves as an explicit reminder that topologically ordered phases emerge always in microscopic systems. The anyon model provides an exact effective low energy description of the system only in an idealised limit of infinite system size and energy gap. The anyons (the Majorana modes at the wire ends) are collective quasiparticle states of underlying more fundamental particles (electrons in the wire), that have also microscopic dynamics of their own. The degeneracy lifting due to finite wave function overlap can be viewed as an anyon-anyon interaction due to microscopics, as discussed in Section 3.4.

\subsection{Quantum computation in nanowire arrays}

\subsubsection{The Majorana qubit}

For all practical purposes, the two localised Majorana modes $\gamma_{1}$ and $\gamma_{2 L}$ at the end of the wire can be viewed as a pair of $\sigma$ anyons of the Ising anyon model (13). We say 'for all practical purposes', because they are not genuine excitations of the system and appear only on the wire ends that are domain walls between the topological phase and the vacuum. This means that they are not free excitations and hence their braiding statistics is only defined projectively, i.e. up to the overall phase [131]. However, since overall phases are irrelevant for quantum computation, they can be treated as Ising anyons.

Adopting this perspective, the occupation of the non-local fermion shared by the two Majorana modes is described by the operator $d^{\dagger} d=\left(1+i \gamma_{1} \gamma_{2 L}\right) / 2$. The state with eigenvalue of $d^{\dagger} d$ being 0 can 
(a)

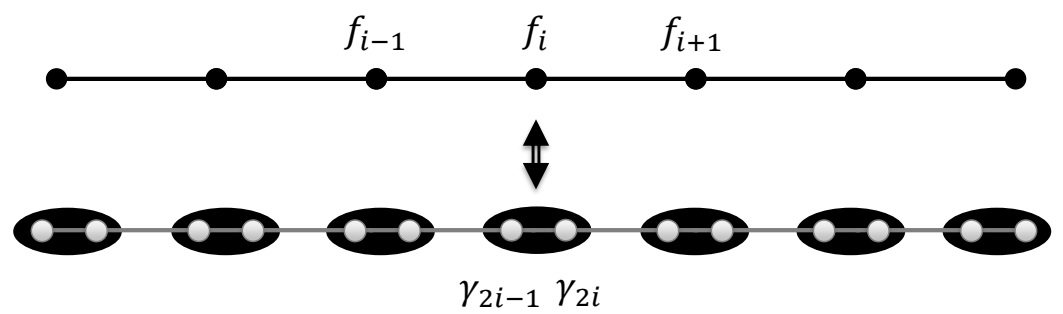

(b)

Trivial phase $(\mu>t)$ :

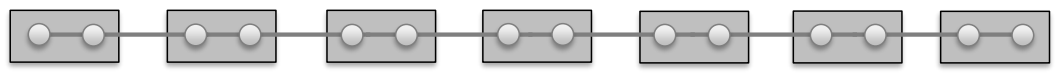

Topological phase $(\mu<t)$ :

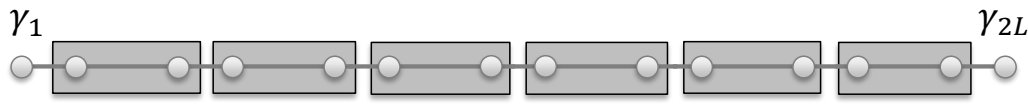

(c)

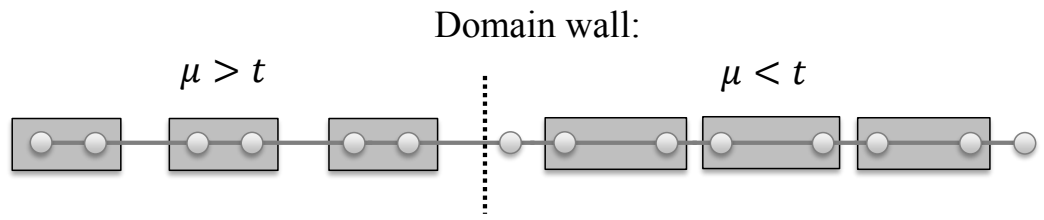

Figure 9: Kitaev's toy model for a $p$-wave paired superconducting wire [55]. (a) The original Hamiltonian (28) in terms of complex fermions $f_{j}$ is defined on a one-dimensional lattice of $L$ sites. When each complex fermion operator is decomposed into two Majoranas by writing $f_{j}=e^{-i \theta / 2}\left(\gamma_{2 j-1}+i \gamma_{2 j}\right) / 2$, the Hamiltonian (29) describes free Majorana fermions on a chain of length $2 L$. (b) When $\mu \gg t,\left|\Delta_{p}\right|$, the system is in the trivial phase described by the Hamiltonian (30), whose unique ground state has all the Majorana modes paired. In the opposing limit, the system is in the topological phase where the Majorana operators $\gamma_{1}$ and $\gamma_{2 L}$ strongly decouple from the Hamiltonian (31). The localized end states constitute a single non-local zero energy fermion mode $d=\left(\gamma_{1}+i \gamma_{2 L}\right) / 2$ and the ground state is thus two-fold degenerate. (c) If a part of the wire is in topological phase and the other part in the trivial phase, then the domain wall separating the phases also binds a localized Majorana mode.

then be identified with the fusion channel $\sigma \times \sigma \rightarrow 1$, while the state with eigenvalue 1 corresponds to $\sigma \times \sigma \rightarrow \psi$. As a result, the two degenerate ground states in the topological phase can be identified with the two fusion channel states

$$
i \gamma_{1} \gamma_{2 L}|\sigma \sigma ; 1\rangle=-|\sigma \sigma ; 1\rangle, \quad i \gamma_{1} \gamma_{2 L}|\sigma \sigma ; \psi\rangle=+|\sigma \sigma ; \psi\rangle
$$

where $|\sigma \sigma ; \psi\rangle=d^{\dagger}|\sigma \sigma ; 1\rangle$. However, as discussed also earlier, these two states cannot form a basis for a qubit, because they belong to different $\psi$-parity sectors. The fermion parity of a wire is described by the operator $\mathscr{P}=\exp \left(i \pi \sum_{j} f_{j}^{\dagger} f_{j}\right)$, which is an exact symmetry of the Hamiltonian 28 . In the twofold degenerate ground state manifold this operator acts as $\mathscr{P}=i \gamma_{1} \gamma_{2 L}$, which means that one can not form coherent superpositions of the two ground states since they belong to different symmetry sectors. To form a qubit one needs two wires, whose ground state manifold contains two states belonging to 
the same parity sector [132, 133, 134]. Choosing the even parity sector $(\mathscr{P}=1)$, the computational basis states can be identified with the fusion channel states $[13)$ as

$$
|0\rangle \equiv|\sigma \sigma ; 1\rangle|\sigma \sigma ; 1\rangle, \quad|1\rangle \equiv|\sigma \sigma ; \psi\rangle|\sigma \sigma ; \psi\rangle,
$$

where the two kets now refer to the fusion channel states of the two wires. One can thus view every wire with Majorana end states as a pair of $\sigma$ anyons created from the vacuum. By adding a third wire, the computational basis of two qubits could be defined precisely as (17).

\subsubsection{Manipulating the Majorana qubit}

The fact that two distinct Majorana wires are required to encode a qubit might seem like posing a problem for manipulating it via braiding. As braiding involves moving the anyons around each other, the obvious problem seems to be that the Majorana modes are stuck at the ends of wires whose position is fixed. However, a wire end is nothing but a domain wall between the topological phase and the vacuum to which the trivial phase is adiabatically connected. Thus Majorana modes exist also at the domain walls that separate the topological phase (a region where $t>|\mu|$ ) from the trivial phase (a region where $t<|\mu|)$, as illustrated in Figure 9. By spatially controlling, say, the chemical potential $\mu$ by gating the wire, even a single physical wire can realize many sections that each contribute a single Majorana wire. By adiabatically changing the chemical potential locally, the domain walls, and thus the Majorana modes bound to them, can in principle be moved along the wires.

To perform braiding one can consider a T-junction of these wires, as illustrated in Figure 10. The junction structure enables exchanging Majorana modes both from same and different topological domains and thereby implement the single qubit Clifford operations (18). It has been explicitly shown [118], that when such exchanges are performed by adiabatically tuning the chemical potential, the resulting non-Abelian Berry phase (23) indeed coincides with the $R$-matrix of the Ising anyon model up to the overall phase. More Majorana qubits can be realized in more complex arrays of junctions.

While moving anyons around by manipulating the chemical potential is the most direct analogue how the implement braiding, it is not the only one to implement the same evolutions in the fusion space. In fact, since tuning the chemical potential involves quenching the system locally into and out of the topological phase to move the domain walls, it can result in unwanted excitations that are sources of error [135, 136]. Several schemes have been devised to implement effective braiding evolutions that do not require actually moving the anyons around. These include switching domains of the wires between Coulomb energy and charging energy dominated regimes enabling Majorana modes to jump between different domain walls [137, 138], controlling the microscopic interactions in a precise manner [139, 140] or the so called measurement-only topological quantum computation, where non-destructive measurements of anyon charges have been shown to simulate arbitrary braid evolutions [141, 142].

\subsubsection{Challenges with Majorana-based topological quantum computation}

In Section 3.4 we discussed general challenges that can affect topological quantum computation. Regarding the presence of unwanted anyons, Majorana wires have a natural source of them due to disorder. Similarly as domain walls can be moved by tuning chemical potential, disorder along the wire, exhibited by a locally random chemical potential, can cause parts of the wire to be accidentally in either the topological or the trivial phase. This causes additional domain walls along the wire that will host additional unaccounted Majorana modes [143, 144]. These will cause leakage out of the 
Transport at junction

(i)

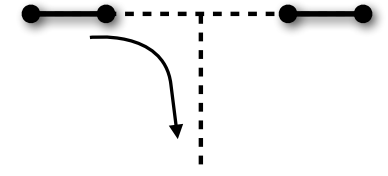

(ii)

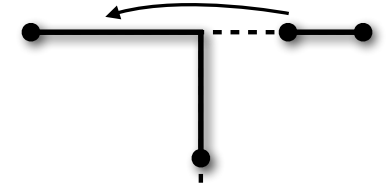

(iii)

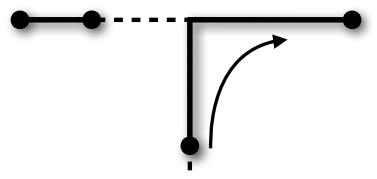

The anyon worldlines

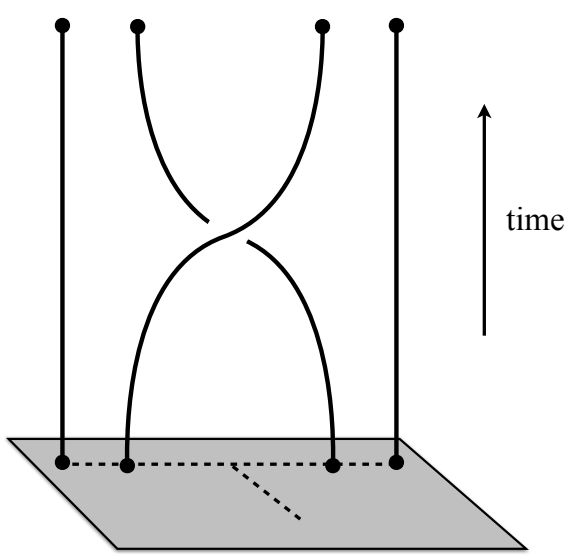

Figure 10: Braiding Majoranas at a T-junction [118]. By locally tuning the chemical potential $\mu$ along the wires, one can change between the topological phase $(|\mu|<t$, solid lines) and the trivial phase ( $|\mu|>t$, dashed lines). The shown sequence (i)-(iii) results in the Majorana modes (dots) bound to the domain walls of different topological domains to be exchanged such that their worldlines are braided. When done adiabatically, the corresponding non-Abelian Berry phase 23 evaluates to the $F^{-1} R F$ braid of the Ising anyon model (up to an overall phase).

computational space and thus the cleanness of the wires is paramount to implement robust topological quantum computation.

Majorana-based schemes can also suffer from the leakage to an external reservoir. The Majorana qubit is protected by the fermion parity that is exact in a closed system [145]. However, the physical schemes to realize Majorana wires require depositing the spin-orbit coupled nanowires on top of a $s$-wave superconductor [56, 57, 52] from which Cooper pairs tunnel into the wire inducing superconductivity. If Cooper pairs can tunnel from the wire, so can Bogoliubov quasiparticles. In other words, the $s$-wave superconductor can also serve as a reservoir of $\psi$ particles breaking the parity conservation. This sets limits on time-scales how fast the quantum computation needs to be performed before such poisoning occurs [146, 147, 148, 149]. Encouragingly, heterostructures for topological nanowires exhibit long poisoning times that should make such errors manageable [150, 151].

Regarding the question of finite temperature, the engineered $p$-wave superconductors may counterintuitively be more robust than intrinsically topologically ordered states. Since Majorana modes are bound to domain walls, they are not spontaneously created by thermal fluctuations. The energy gap protects the localized states on the domain walls from extended states, which suggests that protection by the energy gap should be sufficient. Indeed, studies show that Majorana qubits do tolerate finite temperatures [152, 153], which should thus not pose a fundamental obstacle. 


\section{Outlook}

We have seen that the key ingredients for performing topological quantum computation are: (i) To have access to a system supporting non-Abelian anyons, (ii) to be able to adiabatically move them around each other or simulate such evolutions in a topologically protected manner and (iii) to be able measure their fusion channels. If one were to have access to Fibonacci anyons, then these steps would be sufficient to implement universal quantum computation. Unfortunately, the theoretically proposed states that support these particular types of anyons are very fragile and thus experimentally challenging. Thus the research has focused on simpler types of anyons known as Ising anyons. Their free-fermion counterparts - the Majorana zero modes - are strongly believed to exist, with experimental support in heterostructures of spin-orbit coupled nanowires and normal $s$-wave superconductors [58, 59, 60, 61]. While not universal for quantum computation by purely topological means, they serve as a test bed for the key selling points of topological quantum computation - non-local encoding, protection by energy gap and quantum gates by braiding. If additional non-topological operations are used, then Majorana fermions are able to implement arbitrary quantum gates. Considering that the existence of clear hard energy gaps [154] and the exponential localization of the Majorana end states [62] has already been experimentally verified, there is much hope that the proposed experiments on the braiding properties [118, 119, 138] will also be carried out successfully in these systems.

While being very encouraging, it should be kept in mind that Majorana modes do not provide the full power of topologically protected quantum computation nor is any anyon based scheme a panacea for all the troubles of quantum computation. The hardware level protection they provide is highly desirable, but they still come with their own shortcomings to overcome. Still, considering the open problems faced by non-topological schemes and the rapid progress in preparing and controlling topological states of matter hosting Majorana modes, overcoming these challenges is a fair price to pay for the robustness that comes with topological quantum computation. Ways to go beyond Majorana modes already exist based on the same constructions. Replacing the spin-orbit coupled semiconductor nanowires with edge states of Abelian fractional quantum Hall states can realize parafermion modes that allow for a larger, although still non-universal gate set [155, 156]. While the Read-Rezayi fractional quantum Hall state [29] hosting Fibonacci anyons might just be too fragile, it has been proposed that collective states of parafermion modes can give rise to an analogous state that supports the Fibonacci anyons [80]. Since Abelian fractional quantum Hall states are well established experimentally, it is not too far fetched to imagine that the current technology can be pushed to realize parafermion modes and, stretching the imagination a bit further, perhaps even Fibonacci anyons. Another exotic idea is to employ superconducting nanowires to engineer Ising anyons and 2D and employ defects to promote the Ising anyons for universal quantum computation by topological means [157].

More realistic route might be some midway that enjoys some of the benefits of topological quantum information storing and processing, while not being fully a topological quantum computer as described here. One such scheme is to construct surface codes from Majorana wire based qubits that support Abelian anyons and design fault-tolerant protocols to promote such systems into universal quantum computers [158]. Whatever the route taken, the general principles underlying any topological scheme will be based on the basic operations described in this review.

Funding information JKP would like to acknowledge the support of EPSRC through the grant EP/I038683/1. VTL is supported by the Dahlem Research School POINT fellowship program. 


\section{References}

[1] M. H. Freedman, A. Kitaev, M. J. Larsen and Z. Wang, Topological Quantum Computation, Bull. Amer. Math. Soc. 40, 31 (2003), doi:10.1090/S0273-0979-02-00964-3.

[2] A. Kitaev, Fault-tolerant quantum computation by anyons, Ann. Phys. 303, 2 (2003), doi:10.1016/S0003-4916(02)00018-0

[3] J. M. Leinaas and J. Myrheim, On the theory of identical particles, Nuovo Cimento B 37, 1 (1977), doi:10.1007/BF02727953.

[4] F. Wilczek, Quantum Mechanics of Fractional-Spin Particles, Phys. Rev. Lett. 49, 957 (1982), doi:10.1103/PhysRevLett.49.957.

[5] Y. Aharonov and D. Bohm, Significance of Electromagnetic Potentials in the Quantum Theory, Phys. Rev. 115, 485 (1959), doi:10.1103/PhysRev.115.485.

[6] M. Z. Hasan and C. L. Kane, Topological Insulators, Rev. Mod. Phys. 82, 3045 (2010), doi:10.1103/RevModPhys.82.3045

[7] X.-L. Qi and S.-C. Zhang, Topological insulators and superconductors, Rev. Mod. Phys. 83, 1057 (2011), doi:10.1103/RevModPhys.83.1057.

[8] A. P. Schnyder, S. Ryu, A. Furusaki and A. W. W. Ludwig, Classification of topological insulators and superconductors in three spatial dimensions, Phys. Rev. B 78, 195125 (2008), doi:10.1103/PhysRevB.78.195125.

[9] L. Fu, Topological Crystalline Insulators, Phys. Rev. Lett. 106, 106802 (2011), doi: $10.1103 /$ PhysRevLett.106.106802.

[10] R.-J. Slager, A. Mesaros, V. Juricic and J. Zaanen, The space group classification of topological band insulators, Nat. Phys. 9, 2513 (2013), doi 10.1038/nphys2513.

[11] Z. Wang, A. Alexandradinata, R. J. Cava and B. A. Bernevig, Hourglass Fermions, Nature 532, 189-194 (2016), doi:10.1038/nature17410.

[12] B. Bradlyn, J. Cano, Z. Wang, M.G. Vergniory, C. Felser, R. J. Cava and B. A. Bernevig, Beyond Dirac and Weyl fermions: Unconventional quasiparticles in conventional crystals, Science 353, aaf5037 (2016), doi:10.1126/science.aaf5037.

[13] X. Chen, Z.-C. Gu, Z.-X. Liu, X.-G. Wen, Symmetry protected topological orders in interacting bosonic systems, Science 338, 1604 (2012), doi:10.1126/science.1227224.

[14] X. Chen, Z.-C. Gu, Z.-X. Liu, X.-G. Wen, Symmetry protected topological orders and the group cohomology of their symmetry group, Phys. Rev. B 87, 155114 (2013), doi:10.1103/PhysRevB.87.155114.

[15] Z.-C. Gu and X.-G. Wen, Symmetry-protected topological orders for interacting fermions Fermionic topological nonlinear $\sigma$-models and a special group supercohomology theory, Phys. Rev. B 90, 115141 (2014), doi: 10.1103/PhysRevB.90.115141. 
[16] A. Kitaev and J. Preskill, Topological entanglement entropy, Phys. Rev. Lett. 96, 110404 (2006), doi: 10.1103/PhysRevLett.96.110404.

[17] M. Levin and X.-G. Wen, Detecting Topological Order in a Ground State Wave Function, Phys. Rev. Lett. 96, 110405 (2006), doi 10.1103/PhysRevLett.96.110405.

[18] A. Bullivant and J. K. Pachos, Entropic Topological Invariants in Three Dimensions, Phys. Rev. B 93, 125111 (2016), doi:10.1103/PhysRevB.93.125111.

[19] G. Palumbo and J. K. Pachos, Holographic correspondence in topological superconductors, Ann. Phys. 372, 175 (2016), doi:10.1016/j.aop.2016.05.005.

[20] D. A. Ivanov, Non-Abelian Statistics of Half-Quantum Vortices in p-Wave Superconductors, Phys. Rev. Lett. 86, 268 (2001), doi:10.1103/PhysRevLett.86.268.

[21] X.-G. Wen and Q. Niu, Ground state degeneracy of the FQH states in presence of random potential and on high genus Riemann surfaces, Phys. Rev. B 41, 9377 (1990), doi: $10.1103 /$ PhysRevB.41.9377.

[22] K. von Klitzing, G. Dorda and M. Pepper, New Method for High-Accuracy Determination of the Fine-Structure Constant Based on Quantized Hall Resistance, Phys. Rev. Lett. 45, 494 (1980), doi:10.1103/PhysRevLett.45.494.

[23] D. C. Tsui, H. L. Stormer and A. C. Gossard, Two-Dimensional Magnetotransport in the Extreme Quantum Limit, Phys. Rev. Lett. 48, 1559 (1982), doi:10.1103/PhysRevLett.48.1559.

[24] D. J. Thouless, M. Kohmoto, M. P. Nightingale and M. den Nijs, Quantized Hall Conductance in a Two-Dimensional Periodic Potential, Phys. Rev. Lett. 49, 405 (1982), doi:10.1103/PhysRevLett.49.405.

[25] R. B. Laughlin, Anomalous Quantum Hall Effect: An Incompressible Quantum Fluid with Fractionally Charged Excitations, Phys. Rev. Lett. 50, 1395 (1983), doi:10.1103/PhysRevLett.50.1395.

[26] V. J. Goldman and B. Su, Resonant Tunneling in the Quantum Hall Regime: Measurement of Fractional Charge, Science 267, 1010 (1995), doi:10.1126/science.267.5200.1010.

[27] R. de-Picciotto, M. Reznikov, M. Heiblum, V. Umansky, G. Bunin and D. Mahalu, Direct observation of a fractional charge, Nature 389, 162 (1997), doi:10.1038/38241.

[28] G. Moore and N. Read, Nonabelions in the fractional quantum Hall effect, Nucl. Phys. B 360, 362 (1991), doi:10.1016/0550-3213(91)90407-O.

[29] N. Read and E. Rezayi, Beyond paired quantum Hall states: Parafermions and incompressible states in the first excited Landau level, Phys. Rev. B 59, 8084 (1999), doi:10.1103/PhysRevB.59.8084.

[30] R. L. Willett, L. N. Pfeiffer and K. W. West, Measurement of filling factor 5/2 quasiparticle interference with observation of charge e/4 and e/2 period oscillations, Proc. Nat. Acad. Sci, 106, 8853 (2009), doi:10.1073/pnas.0812599106. 
[31] C. Nayak, S. H. Simon, A. Stern, M. Freedman and S. Das Sarma, Non-Abelian anyons and topological quantum computation, Rev. Mod. Phys. 80, 1083 (2008), doi: $10.1103 /$ RevModPhys.80.1083

[32] N. R. Regnault and B. A. Bernevig, Fractional Chern Insulator, Phys. Rev. X 1, 021014 (2011), doi: 10.1103/PhysRevX.1.021014.

[33] L. Balents, Spin liquids in frustrated magnets, Nature 464, 199-208 (2010), doi:10.1038/nature08917.

[34] J. Reuther, S.-P. Lee and J. Alicea, Classification of spin liquids on the square lattice with strong spin-orbit coupling, Phys. Rev. B 90, 174417 (2014), doi:10.1103/PhysRevB.90.174417.

[35] S. Yan, D. A. Huse and S. R. White, Spin Liquid Ground State of the $S=1 / 2$ Kagome Heisenberg Model, Science 332, 1173-1176 (2011), doi:10.1126/science.1201080.

[36] S. V. Isakov, M. B. Hastings and R. G. Melko, Topological Entanglement Entropy of a BoseHubbard Spin Liquid, Nat. Phys. 7, $772-775$ (2011), doi:10.1038/nphys2036.

[37] H.-C. Jiang, Z. Wang and L. Balents, Identifying Topological Order by Entanglement Entropy, Nat. Phys. 8, 902 (2012), doi:10.1038/nphys2465.

[38] B. Bauer, L. Cincio, B. P. Keller, M. Dolfi, G. Vidal, S. Trebst and A. W. W. Ludwig, Chiral spin liquid and emergent anyons in a Kagome lattice Mott insulator, Nat. Comm. 5, 5137 (2014), doi: $10.1038 /$ ncomms6137.

[39] P. Nataf, M. Lajko, A. Wietek, K. Penc, F. Mila, A, M. Läuchli, Chiral spin liquids in triangular lattice $S U(N)$ fermionic Mott insulators with artificial gauge fields, Phys. Rev. Lett. 117, 167202 (2016), doi:10.1103/PhysRevLett.117.167202.

[40] A. Kitaev, Anyons in an exactly solved model and beyond, Ann. Phys. 321, 2 (2006), doi:10.1016/j.aop.2005.10.005

[41] G. Jackeli and G. Khaliullin, Mott Insulators in the Strong Spin-Orbit Coupling Limit: From Heisenberg to a Quantum Compass and Kitaev Models, Phys. Rev. Lett. 102, 017205 (2009), doi:10.1103/PhysRevLett.102.017205.

[42] Y. Singh, S. Manni, J. Reuther, T. Berlijn, R. Thomale, W. Ku, S. Trebst and P. Gegenwart, Relevance of the Heisenberg-Kitaev model for the honeycomb lattice iridates $\mathrm{A}_{2} \mathrm{IrO}_{3}$, Phys. Rev. Lett. 108, 127203 (2012), doi:10.1103/PhysRevLett.108.127203.

[43] A. Banerjee, C.A. Bridges, J-Q. Yan, A.A. Aczel, L. Li, M.B. Stone, G.E. Granroth, M.D. Lumsden, Y. Yiu, J. Knolle, D.L. Kovrizhin, S. Bhattacharjee, R. Moessner, D.A. Tennant, D.G. Mandrus, S.E. Nagler, Proximate Kitaev Quantum Spin Liquid Behaviour in $\alpha-\mathrm{RuCl}_{3}$, Nat. Mat. 15, 733-740 (2016), doi:10.1038/nmat4604.

[44] M. Hermanns, K. O’Brien and S. Trebst, Weyl spin liquids, Phys. Rev. Lett. 114, 157202 (2015), doi:10.1103/PhysRevLett.114.157202.

[45] K. O'Brien, M. Hermanns and S. Trebst, Classification of gapless $Z_{2}$ spin liquids in threedimensional Kitaev models, Phys. Rev. B 93, 085101 (2016), doi:10.1103/PhysRevB.93.085101. 
[46] M. Levin and X.-G. Wen, String-net condensation: A physical mechanism for topological phases, Phys.Rev. B 71, 045110 (2005), doi:10.1103/PhysRevB.71.045110

[47] N. Read and D. Green, Paired states of fermions in two dimensions with breaking of parity and time-reversal symmetries and the fractional quantum Hall effect, Phys. Rev. B 61, 10267 (2000), doi: 10.1103/PhysRevB.61.10267.

[48] L. Fu and C. L. Kane, Superconducting Proximity Effect and Majorana Fermions at the Surface of a Topological Insulator, Phys. Rev. Lett. 100, 096407 (2008), doi:10.1103/PhysRevLett.100.096407.

[49] J. D. Sau, R. M. Lutchyn, S. Tewari and S. Das Sarma, Generic New Platform for Topological Quantum Computation Using Semiconductor Heterostructures, Phys. Rev. Lett. 104, 040502 (2010), doi:10.1103/PhysRevLett.104.040502.

[50] J. Alicea, Majorana fermions in a tunable semiconductor device, Phys. Rev. B 81, 125318 (2010), doi:10.1103/PhysRevB.81.125318.

[51] T.-P. Choy, J. M. Edge, A. R. Akhmerov and C. W. J. Beenakker, Majorana fermions emerging from magnetic nanoparticles on a superconductor without spin-orbit coupling, Phys. Rev. B 84, 195442 (2011), doi:10.1103/PhysRevB.84.195442.

[52] S. Nadj-Perge, I. K. Drozdov, B. A. Bernevig and A. Yazdani, Proposal for realizing Majorana fermions in chains of magnetic atoms on a superconductor, Phys. Rev. B 88, 020407(R) (2013), doi:10.1103/PhysRevB.88.020407.

[53] M. Duckheim and P. W. Brouwer, Andreev reflection from noncentrosymmetric superconductors and Majorana bound-state generation in half-metallic ferromagnets, Phys. Rev. B 83, 054513 (2011), doi:10.1103/PhysRevB.83.054513.

[54] S.- B. Chung, H.-J. Zhang, X.-L. Qi, and S.-C. Zhang, Topological superconducting phase and Majorana fermions in half-metal/superconductor heterostructures, Phys. Rev. B 84, 060510(R) (2011), doi:10.1103/PhysRevB.84.060510.

[55] A. Kitaev, Unpaired Majorana fermions in quantum wires, Phys.-Usp. 44, 131 (2001), doi: $10.1070 / 1063-7869 / 44 / 10$ S/S29.

[56] R. M. Lutchyn, J. D. Sau, and S. Das Sarma, Majorana Fermions and a Topological Phase Transition in Semiconductor-Superconductor Heterostructures, Phys. Rev. Lett. 105, 077001 (2010), doi:10.1103/PhysRevLett.105.077001.

[57] Y. Oreg, G. Refael, and F. von Oppen, Helical Liquids and Majorana Bound States in Quantum Wires, Phys. Rev. Lett. 105, 177002 (2010), doi:10.1103/PhysRevLett.105.177002.

[58] V. Mourik, K. Zuo, S. M. Frolov, S. R. Plissard, E. P. A. M. Bakkers, and L. P. Kouwenhoven, Signatures of Majorana fermions in hybrid superconductor-semiconductor nanowire devices, Science 336, 1003 (2012), doi:10.1126/science.1222360.

[59] A. Das, Y. Ronen, Y. Most, Y. Oreg, M. Heiblum and H. Shtrikman, Zero-bias peaks and splitting in an Al-InAs nanowire topological superconductor as a signature of Majorana fermions, Nat. Phys. 8, 887-895 (2012), doi:10.1038/nphys2479. 
[60] H. O. H. Churchill, V. Fatemi, K. Grove-Rasmussen, M. T. Deng, P. Caroff, H. Q. Xu and C. M. Marcus, Superconductor-nanowire devices from tunneling to the multichannel regime: Zero-bias oscillations and magnetoconductance crossover, Phys. Rev. B 87, 241401(R) (2013), doi: $10.1103 /$ PhysRevB.87.241401.

[61] S. Nadj-Perge, I. K. Drozdov, J. Li, H. Chen, S. Jeon, J. Seo, A. H. MacDonald, B. A. Bernevig and A. Yazdani, Observation of Majorana fermions in ferromagnetic atomic chains on a superconductor, Science 346, 602 (2014), doi:10.1126/science.1259327.

[62] S. M. Albrecht, A. P. Higginbotham, M. Madsen, F. Kuemmeth, T. S. Jespersen, J. Nygård, P. Krogstrup and C. M. Marcus, Exponential Protection of Zero Modes in Majorana Islands, Nature 531, 206 (2016), doi:10.1038/nature17162.

[63] C. W. J. Beenakker, Search for Majorana fermions in superconductors, Annu. Rev. Con. Mat. Phys. 4, 113 (2013), doi:10.1146/annurev-conmatphys-030212-184337.

[64] J. Alicea, New directions in the pursuit of Majorana fermions in solid state systems, Rep. Prog. Phys. 75, 076501 (2012), doi:10.1088/0034-4885/75/7/076501.

[65] S. R. Elliott and M. Franz, Colloquium: Majorana fermions in nuclear, particle, and solid-state physics, Rev. Mod. Phys. 87, 137 (2015), doi: 10.1103/RevModPhys.87.137

[66] G. Jotzu, M. Messer, R. Desbuquois, M. Lebrat, T. Uehlinger, D. Greif and T. Esslinger, Experimental realisation of the topological Haldane model, Nature 515, 237-240 (2014), doi:10.1038/nature13915.

[67] M. Aidelsburger, M. Lohse, C. Schweizer, M. Atala, J. T. Barreiro, S. Nascimbene, N. R. Cooper, I. Bloch, N. Goldman, Measuring the Chern number of Hofstadter bands with ultracold bosonic atoms, Nat. Phys. 11, 162-166 (2015), doi: 10.1038/nphys3171.

[68] A. Micheli, G. K. Brennen and P. Zoller, A toolbox for lattice spin models with polar molecules, Nat. Phys. 2, 341 - 347 (2006), doi:10.1038/nphys287.

[69] L. Jiang, T. Kitagawa, J. Alicea, A. R. Akhmerov, D. Pekker, G. Refael, J. I. Cirac, E. Demler, M. D. Lukin and P. Zoller, Majorana Fermions in Equilibrium and in Driven Cold-Atom Quantum Wires, Phys. Rev. Lett. 106, 220402 (2011), doi:10.1103/PhysRevLett.106.220402.

[70] S. Diehl, E. Rico, M.A. Baranov and P. Zoller, Topology by Dissipation in Atomic Quantum Wires, Nat. Phys. 7, 971 (2011), doi:10.1038/nphys2106.

[71] A. Bühler, N. Lang, C. V. Kraus, G. Möller, S. D. Huber and H. P. Büchler, Majorana modes and p-wave superfluids for fermionic atoms in optical lattices, Nat. Comm. 5, 4504 (2014), doi:10.1038/ncomms5504.

[72] C.-E. Bardyn and A. Imamoglu, Majorana-like Modes of Light in a One-Dimensional Array of Nonlinear Cavities, Phys. Rev. Lett. 109, 253606 (2012), doi:10.1103/PhysRevLett.109.253606.

[73] J. K. Pachos, W. Wieczorek, C. Schmid, N. Kiesel, R. Pohlner and H. Weinfurter, Revealing anyonic features in a toric code quantum simulation, New J. Phys. 11, 083010 (2009), doi: $10.1088 / 1367-2630 / 11 / 8 / 083010$. 
[74] J.-S. Xu, K. Sun, Y.-J. Han, C.-F. Li, J. K. Pachos and G.-C. Guo, Simulating the exchange of Majorana zero modes with a photonic system, Nat. Comm. 7, 13194 (2016), doi: $10.1038 /$ ncomms 13194 .

[75] E. Witten, Quantum Field Theory and the Jones Polynomial, Commun. Math. Phys. 121, 351 (1989), doi:10.1007/BF01217730.

[76] E. Rowell, R. Stong and Z. Wang, On classification of modular tensor categories, Comm. Math. Phys. 292, 343 (2009), doi:10.1007/s00220-009-0908-z.

[77] S. Trebst, M. Troyer, Z. Wang and A. W.W. Ludwig, A short introduction to Fibonacci anyon models, Prog. Theor. Phys. Supp. 176, 384 (2008), doi:10.1143/PTPS.176.384.

[78] N. E. Bonesteel, L. Hormozi, G. Zikos and S. H. Simon, Braid Topologies for Quantum Computation, Phys. Rev. Lett. 95, 140503 (2005), doi:10.1103/PhysRevLett.95.140503.

[79] M. Baraban, N. E. Bonesteel and S. H. Simon, Resources required for topological quantum factoring, Phys. Rev. A 81, 062317 (2010), doi:10.1103/PhysRevA.81.062317.

[80] R. S. K. Mong, D. J. Clarke, J. Alicea, N. H. Lindner, P. Fendley, C. Nayak, Y. Oreg, A. Stern, E. Berg, K. Shtengel and M. P. A. Fisher, Universal Topological Quantum Computation from a Superconductor-Abelian Quantum Hall Heterostructure, Phys. Rev. X 4, 011036 (2014), doi: 10.1103/PhysRevX.4.011036.

[81] S. Bravyi and A. Kitaev, Fermionic quantum computation, Ann. Phys. 298, 210 (2002), doi:10.1006/aphy.2002.6254.

[82] A. Ahlbrecht, L. S. Georgiev and R. F. Werner, Implementation of Clifford gates in the Ising-anyon topological quantum computer, Phys. Rev. A 79, 032311 (2009), doi: 10.1103/PhysRevA.79.032311.

[83] S. Bravyi and A. Kitaev, Universal quantum computation with ideal Clifford gates and noisy ancillas, Phys. Rev. A 71, 022316 (2005), doi:10.1103/PhysRevA.71.022316.

[84] S. Bravyi, Universal quantum computation with the $v=5 / 2$ fractional quantum Hall state, Phys. Rev. A 73, 042313 (2006), doi:10.1103/PhysRevA.73.042313.

[85] P. Bonderson, D. J. Clarke, C. Nayak and K. Shtengel, Implementing Arbitrary Phase Gates with Ising Anyons, Phys. Rev. Lett. 104, 180505 (2010), doi:10.1103/PhysRevLett.104.180505.

[86] D. J. Clarke and K. Shtengel, Improved phase gate reliability in systems with neutral Ising anyons, Phys. Rev. B 82, 180519(R) (2010), doi:10.1103/PhysRevB.82.180519

[87] T. Karzig, Y. Oreg, G. Refael and M. H. Freedman, A geometric protocol for a robust Majorana magic gate, Phys. Rev. X 6, 031019 (2016), doi:10.1103/PhysRevX.6.031019.

[88] P. Bonderson, Splitting the Topological Degeneracy of Non-Abelian Anyons, Phys. Rev. Lett. 103, 110403 (2009), doi:10.1103/PhysRevLett.103.110403.

[89] M. Cheng, R.M. Lutchyn, V. Galitski and S. Das Sarma, Splitting of Majorana-Fermion Modes due to Intervortex Tunneling in a $p_{x}+i p_{y}$ Superconductor, Phys. Rev. Lett. 103, 107001 (2009), doi:10.1103/PhysRevLett.103.107001. 
[90] M. Baraban, G. Zikos, N. Bonesteel and S.H. Simon, Numerical Analysis of Quasiholes of the Moore-Read Wave Function, Phys. Rev. Lett. 103, 076801 (2009), doi:10.1103/PhysRevLett.103.076801.

[91] V. Lahtinen, Interacting non-Abelian anyons as Majorana fermions in the honeycomb lattice model, New. J. Phys. 13, 075009 (2011), doi:10.1088/1367-2630/13/7/075009

[92] J. R. Wootton, J. Burri, S. Iblisdir and D. Loss, Error Correction for Non-Abelian Topological Quantum Computation, Phys. Rev. X 4, 011051 (2014), doi:10.1103/PhysRevX.4.011051.

[93] V. Lahtinen and J. K. Pachos, Non-Abelian statistics as a Berry phase in exactly solvable models, New J. Phys. 11, 093027 (2009), doi:10.1088/1367-2630/11/9/093027.

[94] M. Cheng, V. Galitski and S. Das Sarma, Nonadiabatic effects in the braiding of non-Abelian anyons in topological superconductors, Phys. Rev. B 84, 104529 (2011), doi:10.1103/PhysRevB.84.104529.

[95] A. Feiguin, S. Trebst, A. W. W. Ludwig, M. Troyer, A. Kitaev, Z. Wang and M. H. Freedman, Interacting Anyons in Topological Quantum Liquids: The Golden Chain, Phys. Rev. Lett. 98, 160409 (2007), doi:10.1103/PhysRevLett.98.160409.

[96] C. Gils, E. Ardonne, S. Trebst, A. W. W. Ludwig, M. Troyer and Z. Wang, Collective States of Interacting Anyons, Edge States, and the Nucleation of Topological Liquids, Phys. Rev. Lett. 103, 070401 (2009), doi:10.1103/PhysRevLett.103.070401.

[97] A. W. W. Ludwig, D. Poilblanc, S. Trebst and M. Troyer, Two-dimensional quantum liquids from interacting non-Abelian anyons, New J. Phys 13, 045014 (2011), doi:10.1088/13672630/13/4/045014.

[98] V. Lahtinen, A. W. W. Ludwig, J.K. Pachos and S. Trebst, Topological liquid nucleation induced by vortex-vortex interactions in Kitaev's honeycomb model, Phys. Rev. B 86, 075115 (2012), doi: $10.1103 /$ PhysRevB.86.075115.

[99] V. Lahtinen, A. W. W. Ludwig and S. Trebst, Perturbed vortex lattices and the stability of nucleated topological phases, Phys. Rev. B 89, 085121 (2014), doi:10.1103/PhysRevB.89.085121.

[100] G. Kells, V. Lahtinen and J. Vala, Kitaev spin models from topological nanowire networks, Phys. Rev. B 89, 075122 (2014), doi:10.1103/PhysRevB.89.075122.

[101] D. Wang, Z. Huang and C. Wu, Fate and remnants of Majorana zero modes in a quantum wire array, Phys. Rev. B 89, 174510 (2014), doi:10.1103/PhysRevB.89.174510.

[102] J. M. Murray and O. Vafek, Majorana bands, Berry curvature, and thermal Hall conductivity in the vortex state of a chiral p-wave superconductor, Phys. Rev. B 92, 134520 (2015), doi:10.1103/PhysRevB.92.134520.

[103] T. Liu and M. Franz, Electronic structure of topological superconductors in the presence of a vortex lattice, Phys. Rev. B 92, 134519 (2015), doi:10.1103/PhysRevB.92.134519.

[104] C. Castelnovo and C. Chamon, Entanglement and topological entropy of the toric code at finite temperature, Phys. Rev. B 76, 184442 (2007), doi:10.1103/PhysRevB.76.184442. 
[105] S. Iblisdir, D. Perez-Garcia, M. Aguado and J. K. Pachos, Thermal States of Anyonic Systems, Nucl. Phys. B 829, 401 (2010), doi:10.1016/j.nuclphysb.2009.11.009

[106] A. Al-Shimary, J. R. Wootton and J. K. Pachos, Lifetime of topological quantum memories in thermal environment, New J. Phys. 15, 025027 (2013), doi:10.1088/1367-2630/15/2/025027

[107] A. Hamma, C. Castelnovo and C. Chamon, Toric-boson model: Toward a topological quantum memory at finite temperature, Phys. Rev. B 79, 245122 (2009), doi: 10.1103/PhysRevB.79.245122.

[108] J. R. Wootton and J. K. Pachos, Bringing order through disorder: Localization of errors in topological quantum memories, Phys. Rev. Lett. 107, 030503 (2011), doi:10.1103/PhysRevLett.107.030503.

[109] F. L. Pedrocchi, A. Hutter, J. R. Wootton and D. Loss, Enhanced thermal stability of the toric code through coupling to a bosonic bath, Phys. Rev. A 88, 062313 (2013), doi: 10.1103/PhysRevA.88.062313.

[110] B. J. Brown, A. Al-Shimary and J. K. Pachos, Entropic Barriers for Two-Dimensional Quantum Memories, Phys. Rev. Lett. 112, 120503 (2014), doi: 10.1103/PhysRevLett.112.120503.

[111] B. J. Brown, D. Loss, J. K. Pachos, C. N. Self, J. R. Wootton, Quantum memories at finite temperature, Rev. Mod. Phys. 88, 45005 (2016), doi:10.1103/RevModPhys.88.045005.

[112] M. V. Berry, Quantal Phase Factors Accompanying Adiabatic Changes, Proc. R. Soc. A 392, 45 (1984), doi $10.1098 /$ rspa.1984.0023.

[113] F. Wilczek and A. Zee, Appearance of Gauge Structure in Simple Dynamical Systems, Phys. Rev. Lett. 52, 2111 (1984), doi:10.1103/PhysRevLett.52.2111.

[114] J. K. Pachos and P. Zanardi, Quantum holonomies for quantum computing, Int. J. Mod. Phys. B 15, 1257 (2001), doi:10.1142/S0217979201004836.

[115] D. Arovas, J.R. Schrieffer and F. Wilczek, Fractional Statistics and the Quantum Hall Effect, Phys. Rev. Lett. 53, 722 (1984), doi:10.1103/PhysRevLett.53.722.

[116] P. Bonderson, V. Gurarie and C. Nayak, Plasma analogy and non-Abelian statistics for Isingtype quantum Hall states, Phys. Rev. B 83, 075303 (2011), doi 10.1103/PhysRevB.83.075303.

[117] N. Read, Non-Abelian adiabatic statistics and Hall viscosity in quantum Hall states and $p_{x}+$ ip $p_{y}$-paired superfluids, Phys. Rev. B 79, 045308 (2009), doi:10.1103/PhysRevB.79.045308.

[118] J. Alicea, Y. Oreg, G. Refael, F. von Oppen, and M.P.A. Fisher, Non-Abelian statistics and topological quantum information processing in $1 D$ wire networks, Nat. Phys. 7, 412 (2011), doi: $10.1038 /$ nphys 1915 .

[119] J. Li, T. Neupert, B. A. Bernevig and A. Yazdani, Manipulating Majorana zero modes on atomic rings with an external magnetic field, Nat. Comm. 7, 10395 (2016), doi:10.1038/ncomms10395.

[120] Y. Tserkovnyak and S. H. Simon, Monte Carlo Evaluation of Non-Abelian Statistics, Phys. Rev. Lett. 90016802 (2003), doi:10.1103/PhysRevLett.90.016802. 
[121] Y.-L. Wu, B. Estienne, N. Regnault, B. A. Bernevig, Braiding Non-Abelian Quasiholes in Fractional Quantum Hall States, Phys. Rev. Lett. 113, 116801 (2014), doi: $10.1103 /$ PhysRevLett.113.116801.

[122] A. T. Bolukbasi and J. Vala, Rigorous calculations of non-Abelian statistics in the Kitaev honeycomb model, New J. Phys. 14, 045007 (2012), doi:10.1088/1367-2630/14/4/045007.

[123] E. Kapit, P. Ginsparg and E. Mueller, Non-Abelian Braiding of Lattice Bosons, Phys. Rev. Lett. 108, 066802 (2012), doi:10.1103/PhysRevLett.108.066802

[124] G. Moore and N. Seiberg: in Superstring 89, edited by M. Green, R. Iengo, S. Randjbar-Daemi, E. Sezgin and A. Stroeminger (World Scientific, Singapore, 1990).

[125] Y. Zhang, T. Grover, A. Turner, M. Oshikawa, and A. Vishwanath, Quasi-particle Statistics and Braiding from Ground State Entanglement, Phys. Rev. B 85, 235151 (2012), doi: $10.1103 /$ PhysRevB.85.235151.

[126] L. Cincio and G. Vidal, Characterizing Topological Order by Studying the Ground States on an Infinite Cylinder, Phys. Rev. Lett. 110, 067208 (2013), doi:10.1103/PhysRevLett.110.067208.

[127] Y. Zhang, T. Grover, and A. Vishwanath, General procedure for determining braiding and statistics of anyons using entanglement interferometry, Phys. Rev. B 91, 035127 (2015), doi:10.1103/PhysRevB.91.035127.

[128] X.-G. Wen, Topological orders and edge excitations in fractional quantum Hall states, Adv. Phys., 44, 405 (1995), doi: 10.1080/00018739500101566.

[129] Li, H. and F. D. M. Haldane, Entanglement Spectrum as a Generalization of Entanglement Entropy: Identification of Topological Order in Non-Abelian Fractional Quantum Hall Effect States, Phys. Rev. Lett. 101, 010504 (2008), doi:10.1103/PhysRevLett.101.010504.

[130] X.-L. Qi, H. Katsura and A. W. W. Ludwig, General Relationship Between the Entanglement Spectrum and the Edge State Spectrum of Topological Quantum States, Phys. Rev. Lett. 108, 196402 (2012), doi:10.1103/PhysRevLett.108.196402.

[131] M. Barkeshli, C.-M. Jian and X.-L. Qi, Genons, twist defects, and projective non-Abelian braiding statistics, Phys. Rev. B 87, 045130 (2013), doi:10.1103/PhysRevB.87.045130.

[132] B. Beri and N. Cooper, Topological Kondo Effect with Majorana Fermions, Phys. Rev. Lett. 109, 156803 (2012), doi:10.1103/PhysRevLett.109.156803

[133] A. Altland and R. Egger, Multiterminal Coulomb-Majorana Junction, Phys. Rev. Lett. 110, 196401 (2013), doi:10.1103/PhysRevLett.110.196401.

[134] S. Plugge, A. Rasmussen, R. Egger and K. Flensberg, Majorana box qubits, New J. Phys 19, 012001 (2017), doi: 10.1088/1367-2630/aa54e1.

[135] G. Kells, D. Sen, J. K. Slingerland, and S. Vishveshwara, Topological blocking in quantum quench dynamics, Phys. Rev. B 89, 235130 (2014), doi:10.1103/PhysRevB.89.235130.

[136] S. Hegde, V. Shivamoggi, S. Vishveshwara and D. Sen, Quench dynamics and parity blocking in Majorana wires, New J. Phys. 17, 053036 (2015), doi:10.1088/1367-2630/17/5/053036. 
[137] J. D. Sau, D. J. Clarke, and S. Tewari, Controlling non-Abelian statistics of Majorana fermions in semiconductor nanowires, Phys. Rev. B 84, 094505 (2011), doi: $10.1103 /$ PhysRevB.84.094505.

[138] D. Aasen, M. Hell, R. V. Mishmash, A. Higginbotham, J. Danon, M. Leijnse, T. S. Jespersen, J. A. Folk, C. M. Marcus, K. Flensberg and J. Alicea, Milestones toward Majorana-based quantum computing, Phys. Rev. X 6, 031016 (2016), doi:10.1103/PhysRevX.6.031016.

[139] T. Hyart, B. van Heck, I. C. Fulga, M. Burrello, A. R. Akhmerov and C. W. J. Beenakker, Flux-controlled quantum computation with Majorana fermions, Phys. Rev. B 88, 035121 (2013), doi: $10.1103 /$ PhysRevB.88.035121.

[140] B. van Heck, A. R. Akhmerov, F. Hassler, M. Burrello, and C. W. J. Beenakker, Coulombassisted braiding of Majorana fermions in a Josephson junction array, New J. Phys. 14, 035019 (2012), doi:10.1088/1367-2630/14/3/035019.

[141] P. Bonderson, M. Freedman and C. Nayak, Measurement-Only Topological Quantum Computation, Phys. Rev. Lett. 101, 010501 (2008), doi:10.1103/PhysRevLett.101.010501.

[142] P. Bonderson, Measurement-only topological quantum computation via tunable interactions, Phys. Rev. B 87, 035113 (2013), doi:10.1103/PhysRevB.87.035113.

[143] P. W. Brouwer, M. Duckheim, A. Romito, and F. von Oppen, Probability Distribution of Majorana End-State Energies in Disordered Wires, Phys. Rev. Lett. 107, 196804 (2011), doi:10.1103/PhysRevLett.107.196804.

[144] A. M. Lobos, R. M. Lutchyn and S. Das Sarma, Interplay of Disorder and Interaction in Majorana Quantum Wires, Phys. Rev. Lett. 109, 146403 (2012), doi:10.1103/PhysRevLett.109.146403

[145] A. R. Akhmerov, Topological quantum computation away from the ground state with Majorana fermions, Phys. Rev. B 82, 020509(R) (2010), doi:10.1103/PhysRevB.82.020509

[146] D. Rainis and D. Loss, Majorana qubit decoherence by quasiparticle poisoning, Phys. Rev. B 85, 174533 (2012), doi:10.1103/PhysRevB.85.174533.

[147] J. C. Budich, S. Walter and B. Trauzettel, Failure of protection of Majorana based qubits against decoherence, Phys. Rev. B 85, 121405 (2012), doi:10.1103/PhysRevB.85.121405.

[148] M. J. Schmidt, D. Rainis, D. Loss, Decoherence of Majorana qubits by noisy gates, Phys. Rev. B 86, 085414 (2012), doi:10.1103/PhysRevB.86.085414.

[149] S.-H. Ho, S.-P. Chao, C.-H. Chou and F.-L. Lin, Decoherence patterns of topological qubits from Majorana modes, New J. Phys. 16, 113062 (2014), doi:10.1088/1367-2630/16/11/113062.

[150] A. P. Higginbotham, S. M. Albrecht, G. Kirsanskas, W. Chang, F. Kuemmeth, P. Krogstrup, T. S. Jespersen, J. Nygard, K. Flensberg and C. M. Marcus, Parity lifetime of bound states in a proximitized semiconductor nanowire, Nat. Phys. 11, 1017 (2015), doi:10.1038/nphys3461.

[151] D. J. van Woerkom, A. Geresdi and L. P. Kouwenhoven, One minute parity lifetime of a NbTiN Cooper-pair transistor, Nat. Phys. 11, 547 (2015), doi:10.1038/nphys3342. 
[152] M. Cheng, R. M. Lutchyn, S. Das Sarma, Topological protection of Majorana qubits, Phys. Rev. B 85, 165124 (2012), doi:10.1103/PhysRevB.85.165124.

[153] L. Mazza, M. Rizzi, M. D. Lukin, and J. I. Cirac, Robustness of quantum memories based on Majorana zero modes, Phys. Rev. B 88, 205142 (2013), doi:10.1103/PhysRevB.88.205142

[154] M. Kjaergaard, F. Nichele, H. J. Suominen, M. P. Nowak, M. Wimmer, A. R. Akhmerov, J. A. Folk, K. Flensberg, J. Shabani, C. J. Palmström and C. M. Marcus, Quantized conductance doubling and hard gap in a two-dimensional semiconductor-superconductor heterostructure, Nat. Commun. 7, 12841 (2016), doi:10.1038/ncomms12841.

[155] N. H. Lindner, E. Berg, G. Refael and A. Stern, Fractionalizing Majorana Fermions: NonAbelian Statistics on the Edges of Abelian Quantum Hall States, Phys. Rev. X 2, 041002 (2012), doi:10.1103/PhysRevX.2.041002.

[156] D. J. Clarke, J. Alicea and K. Shtengel, Exotic non-Abelian anyons from conventional fractional quantum Hall states, Nat. Comm. 4, 1348 (2013), doi:10.1038/ncomms2340

[157] M. Barkeshli and J. D. Sau, Physical Architecture for a Universal Topological Quantum Computer based on a Network of Majorana Nanowires, arXiv:1509.07135.

[158] S. Plugge, L. A. Landau, E. Sela, A. Altland, K. Flensberg and R. Egger, Roadmap to Majorana surface codes, Phys. Rev. B 94, 174514 (2016), doi:10.1103/PhysRevB.94.174514. 